\title{
Search for supersymmetry in events with at least one photon, missing transverse momentum, and large transverse event activity in proton-proton collisions at $\sqrt{s}=13 \mathrm{TeV}$
}

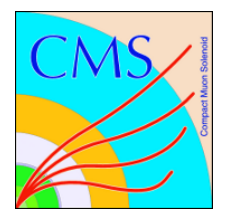

\section{The CMS collaboration}

E-mail: cms-publication-committee-chair@cern.ch

AbStRact: A search for physics beyond the standard model in final states with at least one photon, large transverse momentum imbalance, and large total transverse event activity is presented. Such topologies can be produced in gauge-mediated supersymmetry models in which pair-produced gluinos or squarks decay to photons and gravitinos via shortlived neutralinos. The data sample corresponds to an integrated luminosity of $35.9 \mathrm{fb}^{-1}$ of proton-proton collisions at $\sqrt{s}=13 \mathrm{TeV}$ recorded by the CMS experiment at the LHC in 2016. No significant excess of events above the expected standard model background is observed. The data are interpreted in simplified models of gluino and squark pair production, in which gluinos or squarks decay via neutralinos to photons. Gluino masses of up to $1.50-2.00 \mathrm{TeV}$ and squark masses up to $1.30-1.65 \mathrm{TeV}$ are excluded at $95 \%$ confidence level, depending on the neutralino mass and branching fraction.

KEYwords: Hadron-Hadron scattering (experiments), Supersymmetry, Photon production

ArXIV EPRINT: 1707.06193 


\section{Contents}

1 Introduction 1

2 The CMS detector 2

3 Event reconstruction $\quad 2$

4 Signal models and event simulation 3

$5 \quad$ Event selection and background prediction strategy 4

5.1 Background contribution of events with nongenuine $p_{\mathrm{T}}^{\text {miss }} 4$

5.2 Background contribution from events with electrons 6

$\begin{array}{lll}5.3 & \text { Backgrounds estimated from simulation } & 7\end{array}$

5.4 Validation of the background estimation methods 8

$\begin{array}{llr}6 & \text { Results } & 9\end{array}$

$\begin{array}{lll}7 & \text { Interpretation } & 11\end{array}$

8 Summary 12

$\begin{array}{ll}\text { The CMS collaboration } & 17\end{array}$

\section{Introduction}

The standard model (SM) of particle physics describes elementary particles and their interactions successfully. Nevertheless, fine tuning of fundamental physics parameters is needed to cancel large quantum corrections to the mass term in the Higgs potential [1]. This and other problems of the SM can be addressed by supersymmetry (SUSY) models [2-8], in which a SUSY partner particle is predicted for each SM particle. Gauge-mediated SUSY breaking (GMSB) models [9-15] allow for a natural suppression of flavour violations in the SUSY sector and can give rise to final states with photons and jets [16].

The conservation of $R$ parity [17, 18] implies that SUSY particles are produced in pairs and the lightest SUSY particle (LSP) is stable. If the LSP is neutral and only weakly interacting, it can escape detection, leading to an imbalance of the total observed transverse momentum. In this analysis, $R$-parity conservation is assumed and the LSP is considered to be a nearly massless gravitino $\widetilde{\mathrm{G}}$. The next-to-lightest-supersymmetric particle is assumed to be a gaugino $\widetilde{\chi}_{1}^{0 / \pm}$, which is a mixture of the superpartners of the electroweak gauge bosons and the Higgs bosons. It decays promptly to a SM boson and a gravitino. Both bino- and wino-like neutralinos $\widetilde{\chi}_{1}^{0}$ can decay to a photon and a gravitino; 
wino-like charginos $\widetilde{\chi}_{1}^{ \pm}$decay typically to a $\mathrm{W}$ boson and a gravitino [19]. In this analysis, we assume gauginos are produced in decay chains of primary squarks or gluinos, so the events also contain jets and thus large transverse event activity.

In this paper, a search for physics beyond the standard model (BSM) in final states with at least one photon, large missing transverse momentum, and large total transverse event activity is reported. The data used in this analysis were collected with the CMS detector at the CERN LHC in 2016, and correspond to an integrated luminosity of $35.9 \mathrm{fb}^{-1}$ of proton-proton collisions at a centre-of-mass energy $\sqrt{s}=13 \mathrm{TeV}$. Similar searches yielding no evidence for BSM physics have been performed at lower centre-of-mass energies by CMS [20] with similar and alternative selections [21, 22] and by the ATLAS Collaboration $[23,24]$. The higher $\sqrt{s}$ of this dataset allows us to extend the sensitivity to more massive SUSY particles.

\section{The CMS detector}

The central feature of the CMS apparatus is a superconducting solenoid of $6 \mathrm{~m}$ internal diameter, providing a magnetic field of $3.8 \mathrm{~T}$. Within the solenoid volume are a silicon pixel and strip tracker, a lead tungstate crystal electromagnetic calorimeter (ECAL), and a brass and scintillator hadron calorimeter (HCAL), each composed of a barrel and two endcap sections. The electromagnetic calorimeter consists of 75848 lead tungstate crystals, which provide coverage in pseudorapidity $|\eta|<1.48$ in a barrel region (EB) and $1.48<|\eta|<3.0$ in two endcap regions (EE). Forward calorimeters extend the pseudorapidity coverage provided by the barrel and endcap detectors. Muons are measured in gas-ionization detectors embedded in the steel flux-return yoke outside the solenoid. The jet energy resolution amounts typically to 15,8 , and $4 \%$ at 10,100 , and $1000 \mathrm{GeV}$, respectively, when combining information from the entire detector [25]. A more detailed description of the CMS detector, together with a definition of the coordinate system used and the relevant kinematic variables, can be found in ref. [26].

\section{Event reconstruction}

The particle-flow (PF) algorithm reconstructs and identifies each individual particle with an optimized combination of information from the various elements of the CMS detector [27]. The energy of photons is directly obtained from the ECAL measurement. The energy of electrons is determined from a combination of the electron momentum at the primary interaction vertex as measured by the tracker, the energy of the corresponding ECAL cluster, and the energy sum of all bremsstrahlung photons spatially compatible with originating from the electron track. The momentum of muons is obtained from the curvature of the corresponding track. The energy of charged hadrons is determined from a combination of their momentum measured in the tracker and the matching ECAL and HCAL energy deposits, corrected for zero-suppression effects and for the response function of the calorimeters to hadronic showers. Finally, the energy of neutral hadrons is obtained from the corresponding corrected ECAL and HCAL energies. 
Loose quality criteria with a selection efficiency close to $90 \%$ are applied to photons, based on the shower shape width in $\eta$, the hadronic energy fraction, and the isolation from other particles. To distinguish photons from electrons, photon candidates are not allowed to be associated with pixel seeds. Pixel seeds consist of two or three hits in the pixel detector matching to the hypothetical trajectory from the proton-proton interaction point to the energy cluster in the ECAL, taking into account positively and negatively charged electron hypotheses.

Jets are reconstructed from all PF candidates, clustered by the anti- $k_{\mathrm{T}}$ algorithm $[28$, 29 ] with a distance parameter of 0.4. To reduce the effect of additional proton-proton collisions from the same or adjacent beam crossing (pileup) other than the primary hard scattering process, charged hadrons from vertices not being the primary vertex are excluded. An offset correction is applied to jet energies to take the contribution from pileup interactions into account [30]. The jet momentum is determined as the vector sum of momenta of all PF candidates clustered into the jet. To correct for this, jet energy corrections are applied, derived from simulation and data using multijet, $\gamma+$ jet, and leptonic $\mathrm{Z}+$ jets events.

The missing transverse momentum $\vec{p}_{\mathrm{T}}^{\text {miss }}$ is defined as the negative vector sum of the transverse momenta $p_{\mathrm{T}}$ of all $\mathrm{PF}$ candidates in the event, and its magnitude is denoted by $p_{\mathrm{T}}^{\text {miss }}$. In order to improve the momentum resolution, the jet energy corrections are propagated to $p_{\mathrm{T}}^{\text {miss }}$. The total transverse momentum $H_{\mathrm{T}}^{\gamma}$ is the scalar sum of all jet momenta and the $p_{\mathrm{T}}$ of the leading photon. Only jets with $p_{\mathrm{T}}>30 \mathrm{GeV}$ and $|\eta|<3$ are considered. In addition, if a jet is found within $\Delta R<0.4$ from the leading photon, it is assumed that the jet $p_{\mathrm{T}}$ originates from the photon and the jet $p_{\mathrm{T}}$ is not included in the calculation of $H_{\mathrm{T}}^{\gamma}$.

\section{Signal models and event simulation}

Monte Carlo (MC) generated events are used to study the SM backgrounds, develop and validate the background estimation techniques, and model signal scenarios. To generate $\gamma+$ jet, multijet, $\mathrm{Z}, \mathrm{W}, \mathrm{t} \overline{\mathrm{t}}, \gamma \mathrm{W}, \gamma \mathrm{Z}$, gluino pair, and squark pair events, the MADGRAPH5_aMC@NLO 2.2.2 [31] generator is used at leading-order (LO) accuracy, while the next-to-leading-order (NLO) accuracy is used for $\gamma \mathrm{t} \overline{\mathrm{t}}$ events. The NNPDF3.0 [32] parton distribution functions (PDFs) are used in conjunction with PYтнIA 8.205 or 8.212 [33] with the CUETP8M1 generator tune [34] for simulating parton showering and hadronization. The LO cross sections are used for $\gamma+$ jet events and events comprising solely jets produced through the strong interaction (multijet events). For all other background processes, NLO cross sections are used. The contribution of pileup events is added to the hard scattering process such that the probability of pileup events to occur is the same as that in the data, with on average approximately 23 interactions per bunch crossing.

Gluino and squark pair production cross sections are determined using NLO plus nextto-leading logarithm (NLL) calculations [35]. Four simplified models [36, 37] are considered. The T6gg model, where a first- or second-generation squark-antisquark pair is produced, followed by the (anti)squark decay into an (anti)quark and a neutralino. The neutralino 
decays promptly to a photon and a gravitino, resulting in a final state with two jets, two photons, and missing transverse momentum from the two gravitinos escaping detection. The T6Wg model is similar, except the squarks decay with a probability of $50 \%$ to a quark and a neutralino, and a $50 \%$ probability to decay to a quark and a chargino. The chargino further decays to a $\mathrm{W}$ boson and a gravitino, resulting in signatures with at least two jets, two gravitinos, and two bosons. These two bosons can either be two photons, one photon and one $\mathrm{W}$ boson, or two $\mathrm{W}$ bosons. The T5gg and T5Wg models consist of gluino pair production. For these models, the squark masses are assumed to be much larger than the gluino mass, leading to a three-body decay of the gluino to two jets and a gaugino. For the T5gg model, the gauginos are neutralinos, while for the T5Wg model, the gluino can also decay to jets and a chargino. Branching fractions are assumed to be $100 \%$, except the squark to neutralino branching fraction in the $\mathrm{T} 6 \mathrm{Wg}$ model and the gluino to neutralino decay in the T5Wg model, which are 50\% each. Feynman-like diagrams of these processes are shown in figure 1 .

The CMS detector response is simulated using GEANT4 [38] for SM processes, while for signal events we use the CMS fast simulation [39, 40]. In the latter case, scale factors are applied to account for any differences with respect to the full simulation. Event reconstruction is performed in the same manner as for collision data.

\section{$5 \quad$ Event selection and background prediction strategy}

The high-level trigger system [41] selects events containing at least one photon with $p_{\mathrm{T}}>$ $90 \mathrm{GeV}$ and $|\eta|<2.5$, and $H_{\mathrm{T}}^{\gamma, \mathrm{HLT}}>600 \mathrm{GeV}$, where $H_{\mathrm{T}}^{\gamma, \mathrm{HLT}}$ is defined as the scalar sum of the $p_{\mathrm{T}}$ for all jets passing the kinematic selection used to select jets for the offline $H_{\mathrm{T}}^{\gamma}$ calculation. The trigger does not distinguish between jets and photons. As a result, photons in the event, including the leading photon, are reconstructed as jets and thus included in the calculation of $H_{\mathrm{T}}^{\gamma, \mathrm{HLT}}$. The efficiency for both the photon and the $H_{\mathrm{T}}^{\gamma, \mathrm{HLT}}$ criterion are measured independently, and their product is estimated to be equal to $(96 \pm 4) \%$, where the uncertainty covers variations of the trigger efficiency versus time and versus photon identification variables.

Events are selected if they contain at least one photon with $p_{\mathrm{T}}>100 \mathrm{GeV}$ in the EB with $|\eta|<1.4442$. To reliably predict the background, the photon is not allowed to be parallel or anti-parallel to $\vec{p}_{\mathrm{T}}^{\text {miss }}$ within an azimuthal angle of $\left|\Delta \phi\left( \pm \vec{p}_{\mathrm{T}}^{\text {miss }}, \vec{p}_{\mathrm{T}}^{\gamma}\right)\right|<0.3$. Three high- $p_{\mathrm{T}}^{\text {miss }}$ ranges $(350-450,450-600$, and $\geq 600 \mathrm{GeV})$ and two $H_{\mathrm{T}}^{\gamma}$ selections $(700-2000$ and $\geq 2000 \mathrm{GeV}$ ) give rise to the definition of six search regions. Additional selection criteria are applied to remove events with spurious signals from instrumental noise [42]. Background contributions of multijet, $\gamma+$ jet, $\gamma \mathrm{Z}, \gamma \mathrm{W}, \gamma \mathrm{t} \overline{\mathrm{t}}, \mathrm{W}+$ jets, and $\mathrm{t} \overline{\mathrm{t}}$ events are estimated as described below.

\subsection{Background contribution of events with nongenuine $p_{\mathrm{T}}^{\text {miss }}$}

A small fraction of $\gamma+$ jet events can populate the signal region because of artificial $p_{\mathrm{T}}^{\text {miss }}$ generated by momentum mismeasurement in the detector. Jets have the largest transverse momentum uncertainties, and even though the probability of a large mismeasurement is 

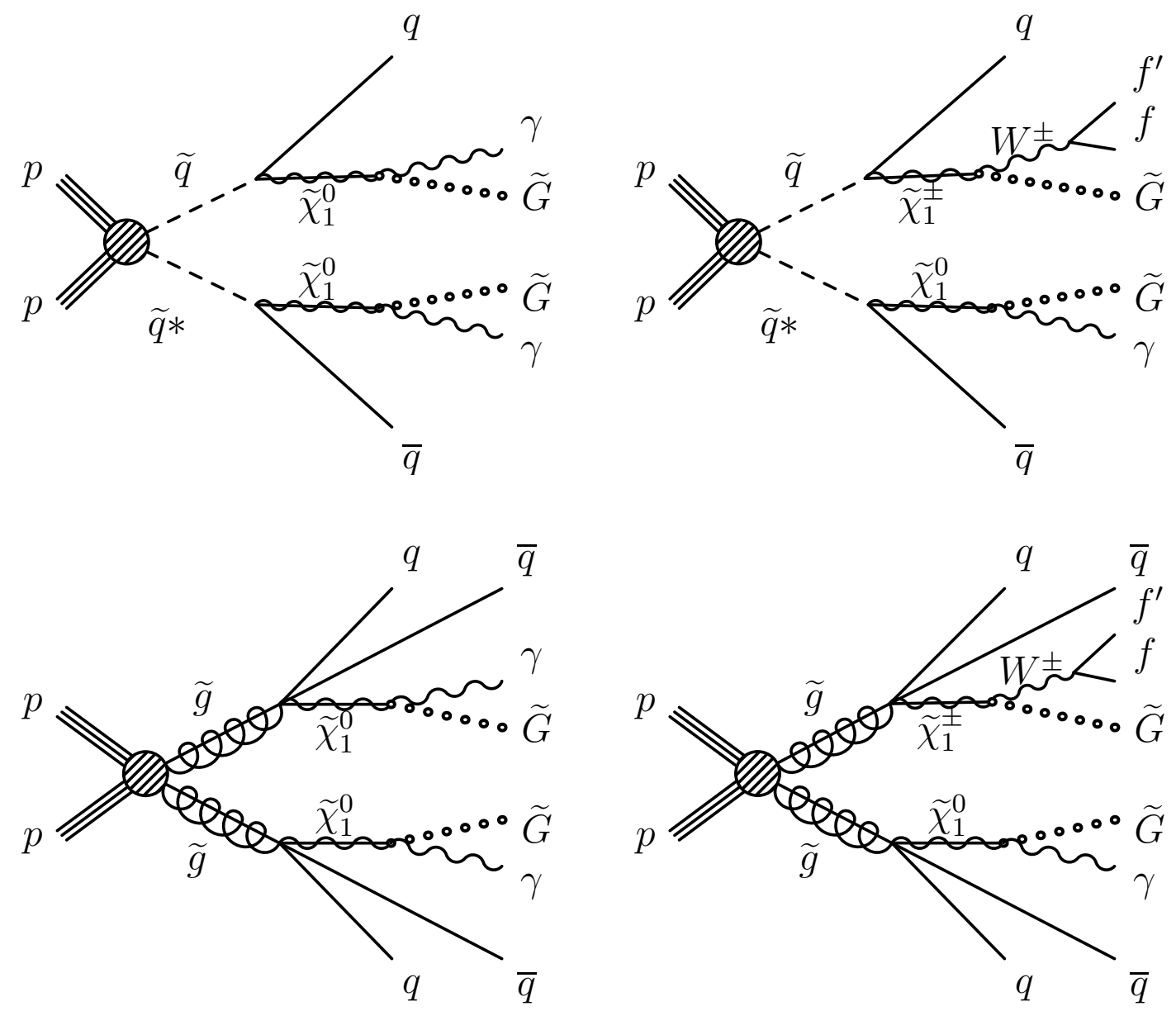

Figure 1. Feynman-like diagrams for the T6gg (top left) and the T5gg (bottom left) processes, and representative Feynman-like diagrams for the $\mathrm{T} 6 \mathrm{Wg}$ (top right) and $\mathrm{T} 5 \mathrm{Wg}$ (bottom right) processes. The $\mathrm{T} 6 \mathrm{Wg}$ and $\mathrm{T} 5 \mathrm{Wg}$ models include also diagrams with either two photons or two W bosons in the final state.

low, the large cross section of the $\gamma+$ jet process leads to a nonnegligible contribution to the search region. Multijet events have an even higher cross section, and contribute to the signal selection if one of the jets is misidentified as a photon. As in $\gamma+$ jet events, nonzero $p_{\mathrm{T}}^{\text {miss }}$ in multijet events is caused by the finite jet momentum resolution.

Estimating these backgrounds from simulation would result in a large uncertainty for two reasons: the large cross section requires a large number of simulated events to obtain a small statistical uncertainty; in addition, small differences between the measured and simulated jet response can lead to large differences at high $p_{\mathrm{T}}^{\text {miss }}$ values between measured and simulated events. A background estimation method based on control samples in data was therefore developed to achieve smaller uncertainties without relying on the simulated jet energy response. We performed this method independently for the low- and high- $H_{\mathrm{T}}^{\gamma}$ selection. The shapes of the $p_{\mathrm{T}}^{\text {miss }}$ distributions in $\gamma+$ jet and multijet events are found 
to be similar, and their normalizations can be extracted from low- $p_{\mathrm{T}}^{\mathrm{miss}}$ events, where no significant signal contribution should be present. This is verified using simulated event samples. We use the shape of the $p_{\mathrm{T}}^{\text {miss }}$ distribution of a multijet event sample as a prediction for events with nongenuine $p_{\mathrm{T}}^{\text {miss }}$.

For the background estimate, the photon control region (CR) is defined by requiring the search selection, but requiring $p_{\mathrm{T}}^{\text {miss }}<100 \mathrm{GeV}$. A jet CR is defined by selecting events with the $H_{\mathrm{T}}^{\gamma}$ criteria only, based on a trigger with only the $H_{\mathrm{T}}^{\gamma, \mathrm{HLT}}$ selection. For low $p_{\mathrm{T}}^{\text {miss }}$ values, the jet $\mathrm{CR}$ is dominated by multijet events, but for large $p_{\mathrm{T}}^{\text {miss }}$ values, $\mathrm{W}(\ell \nu)+$ jets, $\mathrm{Z}(\nu \nu)+$ jets, and $\mathrm{t} \overline{\mathrm{t}}$ events can also contribute. These are subtracted using simulation. The shape of the $p_{\mathrm{T}}^{\text {miss }}$ distribution of $\gamma+$ jet and multijet events in the photon CR is very similar to that in the jet CR.

To correct for residual differences between the two CRs, a correction factor is applied to the $p_{\mathrm{T}}^{\text {miss }}$ values of the jet CR. Studies showed that a constant multiplicative factor leads to the best agreement between the $p_{\mathrm{T}}^{\text {miss }}$ shapes in the two CRs. The factor is chosen such that it minimizes the $\chi^{2}$ between the shapes of the $p_{\mathrm{T}}^{\text {miss }}$ distributions in the two CRs for $p_{\mathrm{T}}^{\text {miss }}<100 \mathrm{GeV}$, and is about $0.90(0.84)$ for the low- (high-) $H_{\mathrm{T}}^{\gamma}$ selection. The uncertainty in this factor is calculated as the quadratic sum of the deviation of the factor from unity and the statistical uncertainty in the $\chi^{2}$ method. The $p_{\mathrm{T}}^{\text {miss }}$ distribution of the jet $\mathrm{CR}$ is then scaled to the $p_{\mathrm{T}}^{\text {miss }}$ distribution of the photon $\mathrm{CR}$ in $p_{\mathrm{T}}^{\text {miss }}<100 \mathrm{GeV}$ to provide an estimate for the background contribution of nongenuine $p_{\mathrm{T}}^{\text {miss }}$ events in the signal selection. Several uncertainties are considered. The uncertainty associated to the shift factor is obtained by multiplying the jet $\mathrm{CR}$ by the factor modulated by its uncertainty. The uncertainty in the normalization is derived from the statistical uncertainty of the photon CR and the jet CR in the $p_{\mathrm{T}}^{\text {miss }}<100 \mathrm{GeV}$ range. The statistical uncertainty assigned to the prediction due to the number of events in the jet CR at high $p_{\mathrm{T}}^{\text {miss }}$ is about as large as the systematic uncertainty.

The method is tested on simulated $\gamma+$ jet and multijet events. The comparison of direct simulation results and the prediction from simulation, using this method, is shown in figure 2. In this figure and the following ones, the rightmost bin includes all events with $p_{\mathrm{T}}^{\text {miss }}>600 \mathrm{GeV}$. The agreement between the two distributions suggests that the method is performing as expected. Further validation is discussed in section 5.4.

\subsection{Background contribution from events with electrons}

Electrons and photons have similar calorimetric response. If no pixel seeds are reconstructed for an electron, it can be misidentified as a photon. In $\mathrm{W}+$ jets or t $\overline{\mathrm{t}}$ processes, electrons are produced in association with neutrinos, so these events tend to also have large $p_{\mathrm{T}}^{\text {miss }}$ and enter the search regions. To estimate the contribution of these processes, a CR with electrons is defined and scaled by the electron-to-photon $(\mathrm{e} \rightarrow \gamma)$ misreconstruction probability.

The electron CR is defined similar to the search selection, except that the photon candidate is required to have pixel seeds, thereby selecting events with electrons. For high $p_{\mathrm{T}}^{\text {miss }}$, this $\mathrm{CR}$ is dominated by $\mathrm{W}$ and $\mathrm{t} \overline{\mathrm{t}}$ events.

The electron-to-photon misreconstruction probability is estimated with the tag-andprobe method using an event sample dominated by $\mathrm{Z} \rightarrow$ ee events, and is $2.7 \%$ for data 

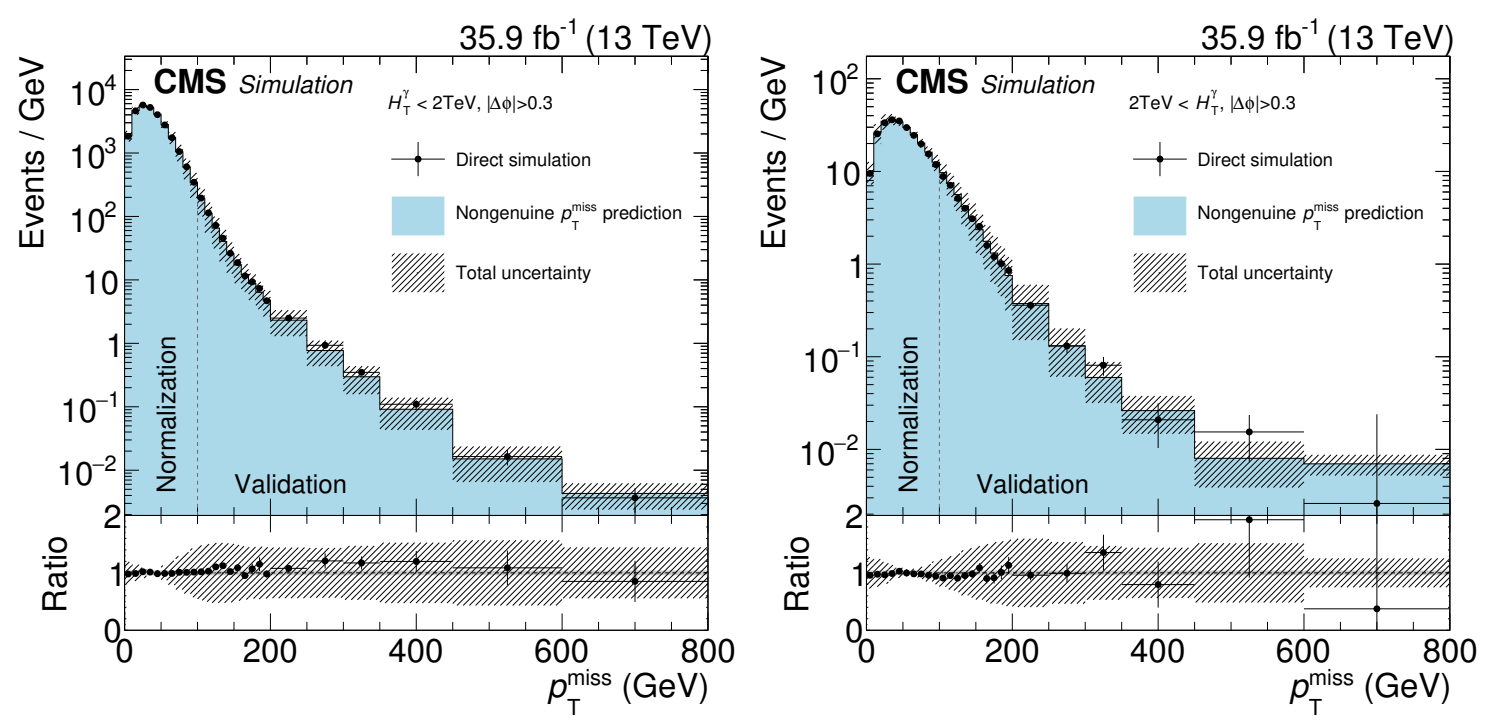

Figure 2. Validation of the nongenuine $p_{\mathrm{T}}^{\text {miss }}$ background estimation method with $\gamma+$ jet and multijet simulations. The direct simulation results are shown as black dots, while the prediction using the jet $\mathrm{CR}$ is shown as light blue histogram. The total uncertainty of the prediction is presented as shaded area. The bottom panel shows the ratio of the direct simulation to the prediction. The low- (high-) $H_{\mathrm{T}}^{\gamma}$ selection is shown on the left (right). The number of events corresponds to the expectation in data for an integrated luminosity of $35.9 \mathrm{fb}^{-1}$. The rightmost bin includes all events with $p_{\mathrm{T}}^{\text {miss }}>600 \mathrm{GeV}$.

and $1.5 \%$ for simulation. For the prediction in data, the probability measured with data is used, while for the validation in simulation, the probability measured with simulated events is used. To account for differences between the misreconstruction rate determined from the $\mathrm{Z}$ boson resonance and the $\mathrm{W}$ boson dominated electron $\mathrm{CR}$ with high $p_{\mathrm{T}}^{\mathrm{miss}}$ and high $H_{\mathrm{T}}^{\gamma}$, a systematic uncertainty of $30 \%$ is applied to the misreconstruction rate. The size of the uncertainty is based on studies of the variation of the misreconstruction probability versus various kinematic and geometric quantities in data and simulation.

The background estimation method is tested on simulated $\mathrm{W}+$ jets and $\mathrm{t} \overline{\mathrm{t}}$ events. The direct simulation of electrons reconstructed as photons is compared to the electron CR, scaled by the electron-to-photon misreconstruction probability as shown in figure 3 , but including also low $p_{\mathrm{T}}^{\text {miss }}$ events. The agreement in the search regions suggests that the method is performing as expected.

\subsection{Backgrounds estimated from simulation}

Also contributing to the search region are the processes $\gamma \mathrm{W}(\ell \nu), \gamma \mathrm{Z}(\nu \nu)$, and $\gamma \mathrm{t} \overline{\mathrm{t}}$, which are estimated using simulation. Simulated events with electrons reconstructed as photons passing the event selection are omitted since they are estimated using data. The photon in the event can be produced in the hard scattering or in the shower, either as initial- (ISR) or final-state radiation, or as a jet misreconstructed as a photon. Events are simulated with and without a photon in the hard scattering process, and the overlap between the samples is removed. The reconstruction and identification efficiencies for photons are measured in 

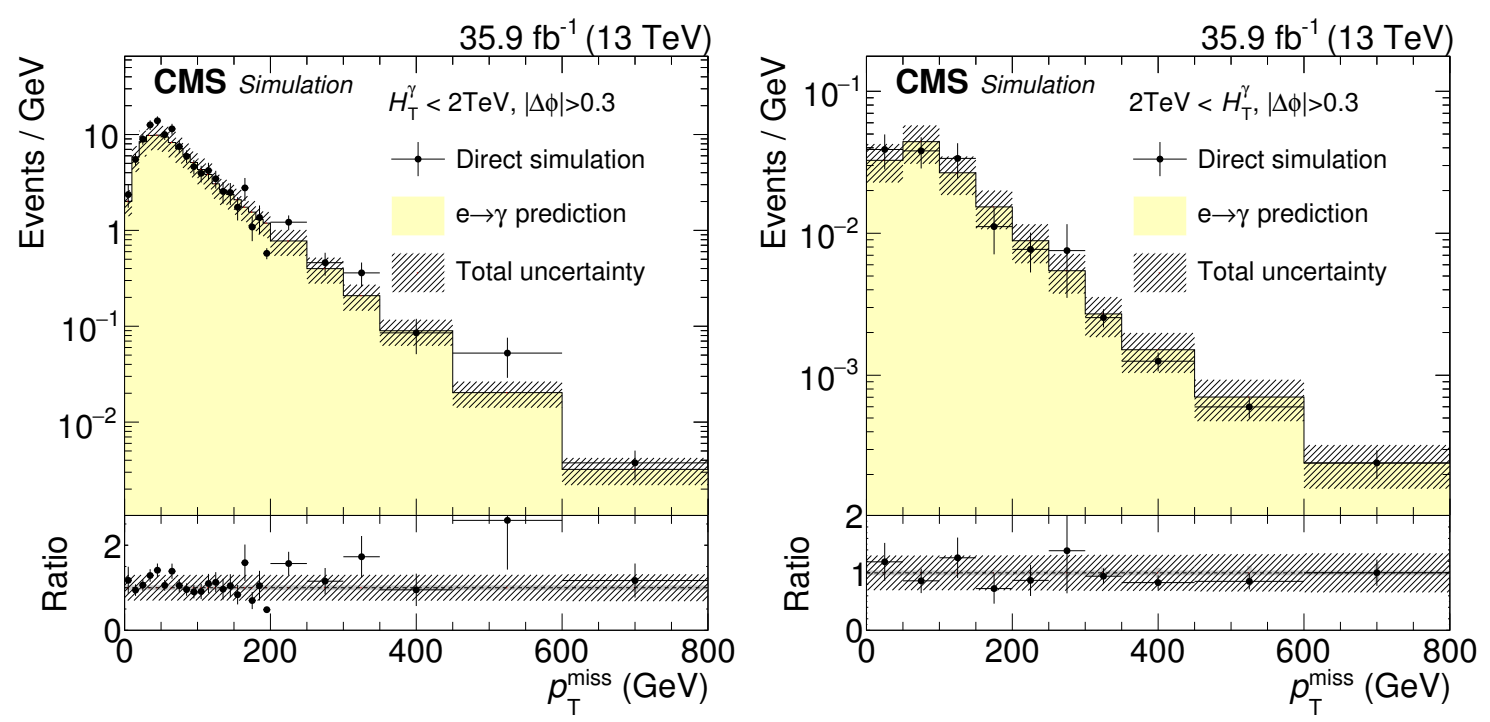

Figure 3. Validation of the background estimation method for electrons misreconstructed as photons using $\mathrm{W}+$ jets and $\mathrm{t} \overline{\mathrm{t}}$ simulation. The low- (high-) $H_{\mathrm{T}}^{\gamma}$ selection is shown on the left (right). The number of events corresponds to the expectation in data for an integrated luminosity of $35.9 \mathrm{fb}^{-1}$. The rightmost bin includes all events with $p_{\mathrm{T}}^{\text {miss }}>600 \mathrm{GeV}$.

$\mathrm{Z} \rightarrow$ ee and $\mathrm{Z} \rightarrow \mu \mu \gamma$ data and simulation. The ratio of these efficiencies is consistent with unity and has an uncertainty of about $3 \%$. Simulated events are weighted by the ratio of the efficiencies, and the uncertainty is propagated to the event yield. The NLO cross sections are used, and several uncertainties are considered, with their relative uncertainties given here in parentheses: factorization and renormalization scales (16-27\%), PDFs (5$10 \%$ ) [43], contribution of pileup events $(0.2-6 \%)$, trigger efficiency $(4 \%)$, jet resolution and energy scales (2-20\%), integrated luminosity (2.5\%) [44], and statistical uncertainty of the simulated samples (4-47\%). For the study of the renormalization and factorization scale uncertainties, variations up and down by a factor of two with respect to the nominal values of the scales are considered. The maximum difference in the yields with respect to the nominal case is used as the uncertainty. The pileup uncertainty corresponds to the variation of the number of predicted events if the total inelastic proton-proton cross section is shifted by $\pm 5 \%$.

\subsection{Validation of the background estimation methods}

In addition to the validation of the background estimation methods with simulated events, the methods are also validated using data from two mutually exclusive event selections. The first validation region is defined with noncentral photons. Instead of the photon being reconstructed in the $\mathrm{EB}$, the leading photon must be reconstructed in the range $1.6<|\eta|<$ 2.5. This is not the full range of the $\mathrm{EE}$, but in this range the background contribution from electrons reconstructed as photons is similar to the one in the EB search region. Highmass gluinos and squarks tend to decay more centrally, leaving the EE validation region essentially free of potential signal events. The same methods as for the EB search regions 

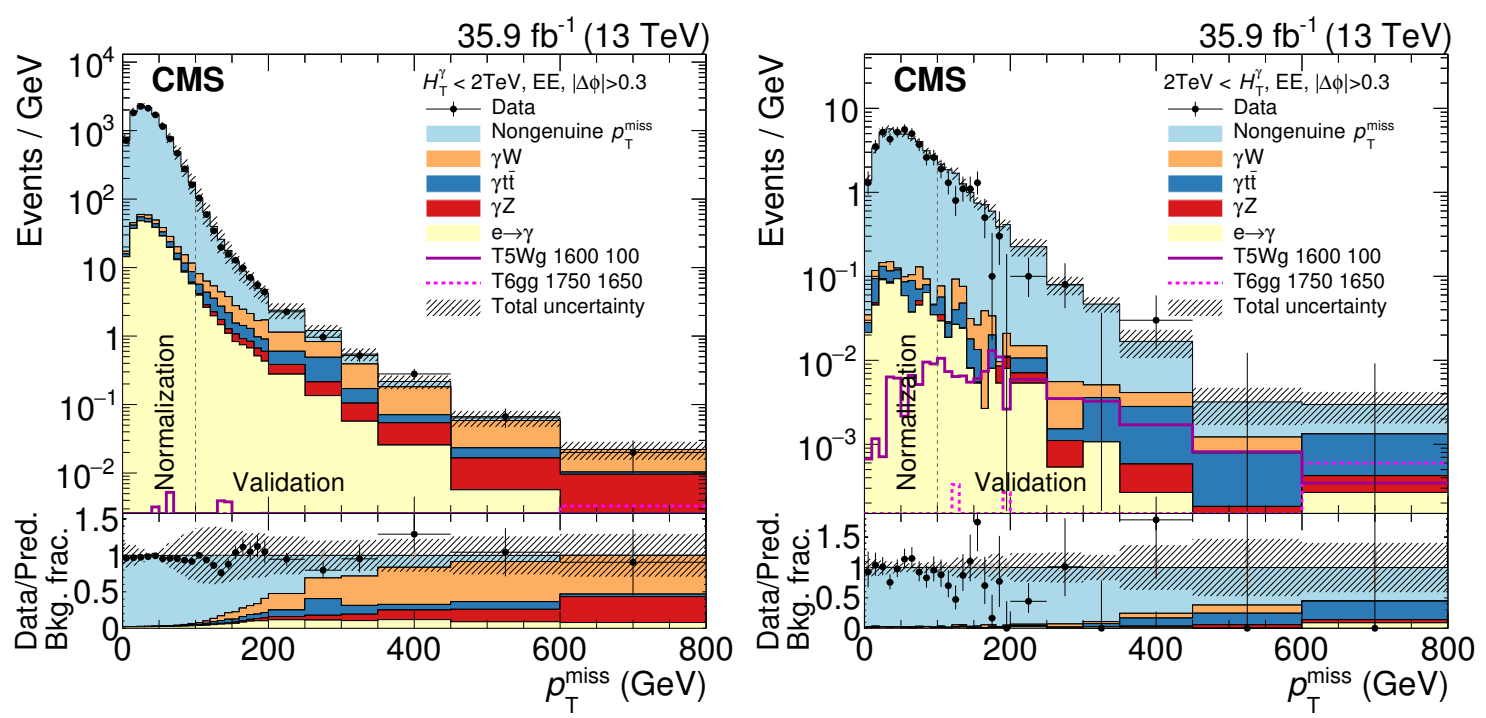

Figure 4. Validation of the background estimation methods with photons reconstructed in the EE. The expectation for the $\mathrm{T} 5 \mathrm{Wg}$ signal scenario with a gluino mass of $1600 \mathrm{GeV}$ and a gaugino mass of $100 \mathrm{GeV}$ and the T6gg signal scenario with a squark mass of $1750 \mathrm{GeV}$ and a neutralino mass of $1650 \mathrm{GeV}$ are shown. The low- (high-) $H_{\mathrm{T}}^{\gamma}$ selection is shown on the left (right). Below the $p_{\mathrm{T}}^{\mathrm{miss}}$ distributions, the data divided by the background prediction are shown as black dots, and the relative background components are shown as coloured areas. The rightmost bin includes all events with $p_{\mathrm{T}}^{\text {miss }}>600 \mathrm{GeV}$.

are applied, and the resulting distributions are shown in figure 4. The $p_{\mathrm{T}}^{\mathrm{miss}}$ distributions of two signal models are displayed as well. In the low- $H_{\mathrm{T}}^{\gamma}$ region and for large $p_{\mathrm{T}}^{\text {miss }}$ of the high- $H_{\mathrm{T}}^{\gamma}$ region, the observed number of events agrees with the prediction. The second validation region is similar to the search regions with photons reconstructed in the EB, with $100<p_{\mathrm{T}}^{\text {miss }}<350 \mathrm{GeV}$, which is orthogonal to both the region used to normalize the multijet background $\left(p_{\mathrm{T}}^{\text {miss }}<100 \mathrm{GeV}\right)$ as well as the signal regions $\left(p_{\mathrm{T}}^{\text {miss }}>350 \mathrm{GeV}\right)$, and is shown in figure 5 . Good agreement is observed in this validation region as well.

\section{Results}

The predicted number of SM background events, the expected signal yield for two signal scenarios and the number of observed events in data are shown in figure 5 and table 1. The uncertainties (including the uncertainties for the signal models) are presented in table 2. The low- $H_{\mathrm{T}}^{\gamma}$ search regions are dominated by $\gamma \mathrm{W}$ events and are sensitive to signal models with low squark or gluino masses. The high- $H_{\mathrm{T}}^{\gamma}$ search regions are dominated by background with nongenuine $p_{\mathrm{T}}^{\text {miss }}$ and have larger sensitivity to models with high gluino or squark masses and low gaugino masses. Overall, the number of observed events is in agreement with the prediction. The second search bin in both the low- and high- $H_{\mathrm{T}}^{\gamma}$ regions shows an excess with local significance of 1.9 and 2.7 standard deviations $(\sigma)$, respectively. In the highest $p_{\mathrm{T}}^{\text {miss }}$ bins, which are more sensitive for most signal scenarios, the number of observed events is compatible with the background expectation. 

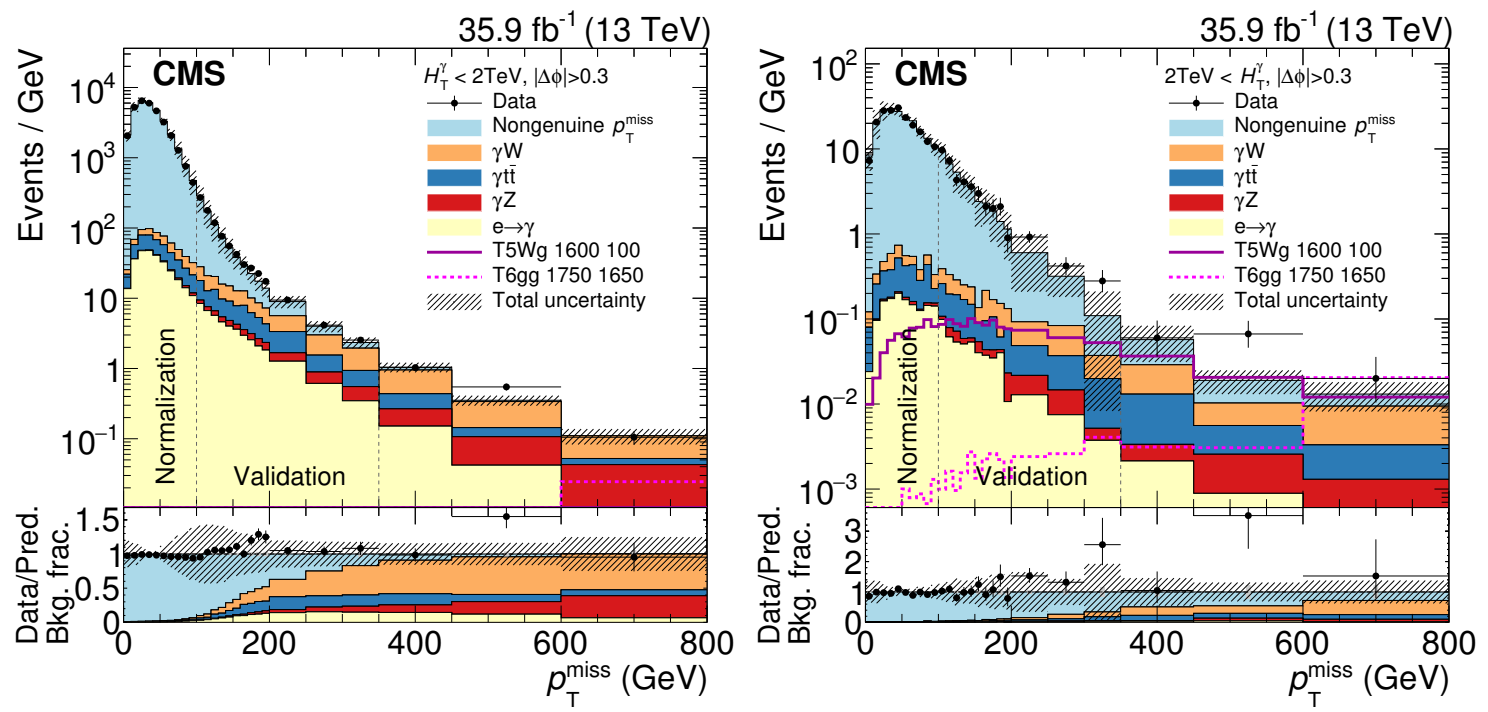

Figure 5. Observed data compared to the background prediction. The expectation for the T5Wg signal scenario with a gluino mass of $1600 \mathrm{GeV}$ and a gaugino mass of $100 \mathrm{GeV}$ and the T6gg signal scenario with a squark mass of $1750 \mathrm{GeV}$ and a neutralino mass of $1650 \mathrm{GeV}$ are shown. The low(high-) $H_{\mathrm{T}}^{\gamma}$ selection is shown on the left (right). Below the $p_{\mathrm{T}}^{\text {miss }}$ distributions, the data divided by the background prediction are shown as black dots, and the relative background components are shown as coloured areas. The last three bins in each plot correspond to the search regions. The rightmost bin includes all events with $p_{\mathrm{T}}^{\text {miss }}>600 \mathrm{GeV}$.

\begin{tabular}{|c|c|c|c|c|c|c|}
\hline $\begin{array}{l}H_{\mathrm{T}}^{\gamma}(\mathrm{GeV}) \\
p_{\mathrm{T}}^{\text {miss }}(\mathrm{GeV})\end{array}$ & $(350,450)$ & $\begin{array}{l}<2000 \\
(450,600)\end{array}$ & $>600$ & $(350,450)$ & $\begin{array}{c}>2000 \\
(450,600)\end{array}$ & $>600$ \\
\hline Nongenuine $p_{\mathrm{T}}^{\text {miss }}$ & $9.6_{-}^{+} \begin{array}{r}11.1 \\
9.6\end{array}$ & $2.2_{-2.2}^{+5.5}$ & $<0.1$ & $2.83 \pm 2.51$ & $1.31 \pm 0.74$ & $0.73_{-0.73}^{+0.86}$ \\
\hline$\gamma \mathrm{W}$ & $51.3 \pm 9.7$ & $29.1 \pm 5.5$ & $11.6 \pm 2.5$ & $1.58 \pm 0.58$ & $0.70 \pm 0.37$ & $1.23 \pm 0.43$ \\
\hline$\gamma \mathrm{t} \overline{\mathrm{t}}$ & $17.1 \pm 5.4$ & $5.6 \pm 2.6$ & $1.9 \pm 0.4$ & $0.97 \pm 0.38$ & $0.45 \pm 0.29$ & $0.40 \pm 0.22$ \\
\hline$\gamma \mathrm{Z}$ & $11.5 \pm 2.4$ & $9.7 \pm 1.8$ & $7.1 \pm 1.4$ & $0.12 \pm 0.07$ & $0.25 \pm 0.11$ & $0.21 \pm 0.10$ \\
\hline $\mathrm{e} \rightarrow \gamma$ & $15.1 \pm 4.6$ & $6.3 \pm 1.9$ & $1.4 \pm 0.5$ & $0.21 \pm 0.10$ & $0.13 \pm 0.07$ & $0.05 \pm 0.04$ \\
\hline Total bkg. & $104.6 \pm 16.5$ & $53.0 \pm 8.6$ & $22.0 \pm 3.0$ & $5.72 \pm 2.60$ & $2.84 \pm 0.89$ & $2.62 \pm 0.99$ \\
\hline Data & 103 & 82 & 21 & 6 & 10 & 4 \\
\hline T5Wg 1600100 & $0.4 \pm 0.1$ & $0.8 \pm 0.1$ & $0.7 \pm 0.1$ & $3.66 \pm 0.40$ & $3.09 \pm 0.40$ & $2.41 \pm 0.32$ \\
\hline T6gg $\quad 1750 \quad 1650$ & $0.5 \pm 0.1$ & $0.8 \pm 0.1$ & $4.9 \pm 0.4$ & $0.31 \pm 0.04$ & $0.46 \pm 0.07$ & $4.12 \pm 0.32$ \\
\hline
\end{tabular}

Table 1. Observed data compared to the background prediction and the expected signal yields for two signal scenarios. The expectations are given for the $\mathrm{T} 5 \mathrm{Wg}$ signal scenario with a gluino mass of $1600 \mathrm{GeV}$ and a gaugino mass of $100 \mathrm{GeV}$ and the T6gg signal scenario with a squark mass of $1750 \mathrm{GeV}$ and a neutralino mass of $1650 \mathrm{GeV}$. The quadratic sum of statistical and systematical uncertainties is given. Only experimental uncertainties for the signal model are stated. 


\begin{tabular}{|l|cc|}
\hline & \multicolumn{2}{|c|}{ Relative uncertainty (\%) } \\
background & signal \\
\hline Nongenuine $p_{\mathrm{T}}^{\text {miss }}$ & $14-250$ \\
$\mathrm{e} \rightarrow \gamma$ & 30 \\
\hline Integrated luminosity & 2.5 & \\
Photon scale factors & 2 & 2.5 \\
Trigger & 4 & 4 \\
PDFs & $5-10$ & \\
Renormalization/factorization scales & $16-27$ & $0-1$ \\
Jet energy scale and resolution & $2-20$ & $1-6$ \\
Pileup & $0.2-6$ & $0.2-10$ \\
ISR & & $0-10$ \\
Fast simulation $p_{\mathrm{T}}^{\text {miss }}$ modelling & & $0.5-6$ \\
\hline
\end{tabular}

Table 2. Systematic uncertainties for background determined from control samples in data (first two rows) and simulation (all other rows). If two values are given, the first one is for simulated SM backgrounds, while the latter is for simulated signal. The PDF and scale uncertainties for the signal simulation affect the shape only, as the uncertainty in the rate is already considered in the overall cross section uncertainty [35].

\section{Interpretation}

The systematic uncertainties of the nongenuine $p_{\mathrm{T}}^{\text {miss }}$ background are fully correlated within the high- and low- $H_{\mathrm{T}}^{\gamma}$ selections, and are described in section 5.1. The systematic uncertainty in the electron misidentification background is fully correlated for all search regions, as are most uncertainties in the simulated backgrounds described in section 5.3.

To improve on the signal simulation of the multiplicity of additional jets from ISR, simulated signal events are reweighted based on the number of ISR jets $\left(N_{\mathrm{J}}^{\mathrm{ISR}}\right)$ so as to make the jet multiplicity in simulated $t \bar{t}$ samples agree with that in data. The reweighting factors vary between 0.92 and 0.51 for $N_{\mathrm{J}}^{\mathrm{ISR}}$ between 1 and 6 . We take one half of the deviation from unity as the systematic uncertainty in these reweighting factors, correlated between all search regions. The renormalization and factorization scales, and PDF uncertainties in the cross sections for signal simulation are taken from ref. [35]. To estimate the influence of pileup in signal events, the selection is done with a high and a low number of additional interactions. The difference in selection efficiency is taken as a systematic uncertainty. Since all physics objects are included in the computation of $p_{\mathrm{T}}^{\text {miss }}$, it can be difficult to describe accurately within the CMS fast simulation. The $p_{\mathrm{T}}^{\text {miss }}$ of the models considered, however, is dominated by the missing momentum carried away by the gravitons and not by the modelling of resolution effects. An additional systematic uncertainty of between 0.5 and $6 \%$ is assigned by calculating the mean difference between the reconstructed and generated $p_{\mathrm{T}}^{\text {miss }}$. A summary of the uncertainties can be found in table 2 .

The results are interpreted in terms of the simplified models introduced in section 4 . The $95 \%$ confidence level (CL) upper limits on the SUSY cross section are calculated with 
the $\mathrm{CL}_{\mathrm{S}}$ criterion [45, 46] using the LHC-style profile likelihood ratio as test statistic [47] evaluated in the asymptotic approximation [48]. Log-normal nuisance parameters are used to describe the systematic uncertainties. The observed upper limits on cross sections, exclusion contours, and expected exclusion contours are shown in figure 6. More stringent limits can be set on models with two photons, since the probability that at least one photon is reconstructed is higher. In this case, for high gaugino masses, squarks up to $1650 \mathrm{GeV}$ and gluinos up to $2000 \mathrm{GeV}$ can be excluded, while for the $\mathrm{T} 6 \mathrm{Wg}$ and $\mathrm{T} 5 \mathrm{Wg}$ scenarios, squarks up to $1550 \mathrm{GeV}$ and gluinos up to $1900 \mathrm{GeV}$ can be excluded for high gaugino masses. The acceptance drops for low neutralino masses, since more energy is transferred to jets, leaving less energy available for the photon and the gravitinos, and therefore resulting in a lower value of $p_{\mathrm{T}}^{\text {miss }}$. If the chargino mass is close to the $\mathrm{W}$ boson mass, less momentum is transferred to the gravitino, leading to smaller $p_{\mathrm{T}}^{\text {miss }}$ values and, therefore, lower sensitivity. This yields a squark mass exclusion of 1500 and $1300 \mathrm{GeV}$ for the T6gg and T6Wg model, respectively, and a gluino mass exclusion of 1750 and $1500 \mathrm{GeV}$ for the $\mathrm{T} 5 \mathrm{gg}$ and $\mathrm{T} 5 \mathrm{Wg}$ model, respectively. For squark pair production, the mass exclusion is determined assuming eight mass-degenerate squark states, corresponding to the SUSY partners of the left- and right-handed $\mathrm{u}, \mathrm{d}, \mathrm{s}$, and c quarks.

\section{Summary}

A search for physics beyond the standard model (SM) in final states with at least one photon, large missing transverse momentum, and large total transverse event activity has been presented using data corresponding to an integrated luminosity of $35.9 \mathrm{fb}^{-1}$ of protonproton collisions at $\sqrt{s}=13 \mathrm{TeV}$ recorded by the CMS experiment at the LHC in 2016. The $\mathrm{SM}$ background is estimated from data and simulation, and is validated in several control regions. No significant signs of new physics beyond the SM are found, and the data are interpreted in simplified models motivated by gauge-mediated supersymmetry breaking. Gluino masses up to $1.50-2.00 \mathrm{TeV}$ and squark masses up to $1.30-1.65 \mathrm{TeV}$ are excluded at $95 \%$ confidence level, depending on the neutralino mass and mixture.

\section{Acknowledgments}

We congratulate our colleagues in the CERN accelerator departments for the excellent performance of the LHC and thank the technical and administrative staffs at CERN and at other CMS institutes for their contributions to the success of the CMS effort. In addition, we gratefully acknowledge the computing centres and personnel of the Worldwide LHC Computing Grid for delivering so effectively the computing infrastructure essential to our analyses. Finally, we acknowledge the enduring support for the construction and operation of the LHC and the CMS detector provided by the following funding agencies: BMWFW and FWF (Austria); FNRS and FWO (Belgium); CNPq, CAPES, FAPERJ, and FAPESP (Brazil); MES (Bulgaria); CERN; CAS, MoST, and NSFC (China); COLCIENCIAS (Colombia); MSES and CSF (Croatia); RPF (Cyprus); SENESCYT (Ecuador); MoER, ERC IUT, and ERDF (Estonia); Academy of Finland, MEC, and HIP (Finland); 

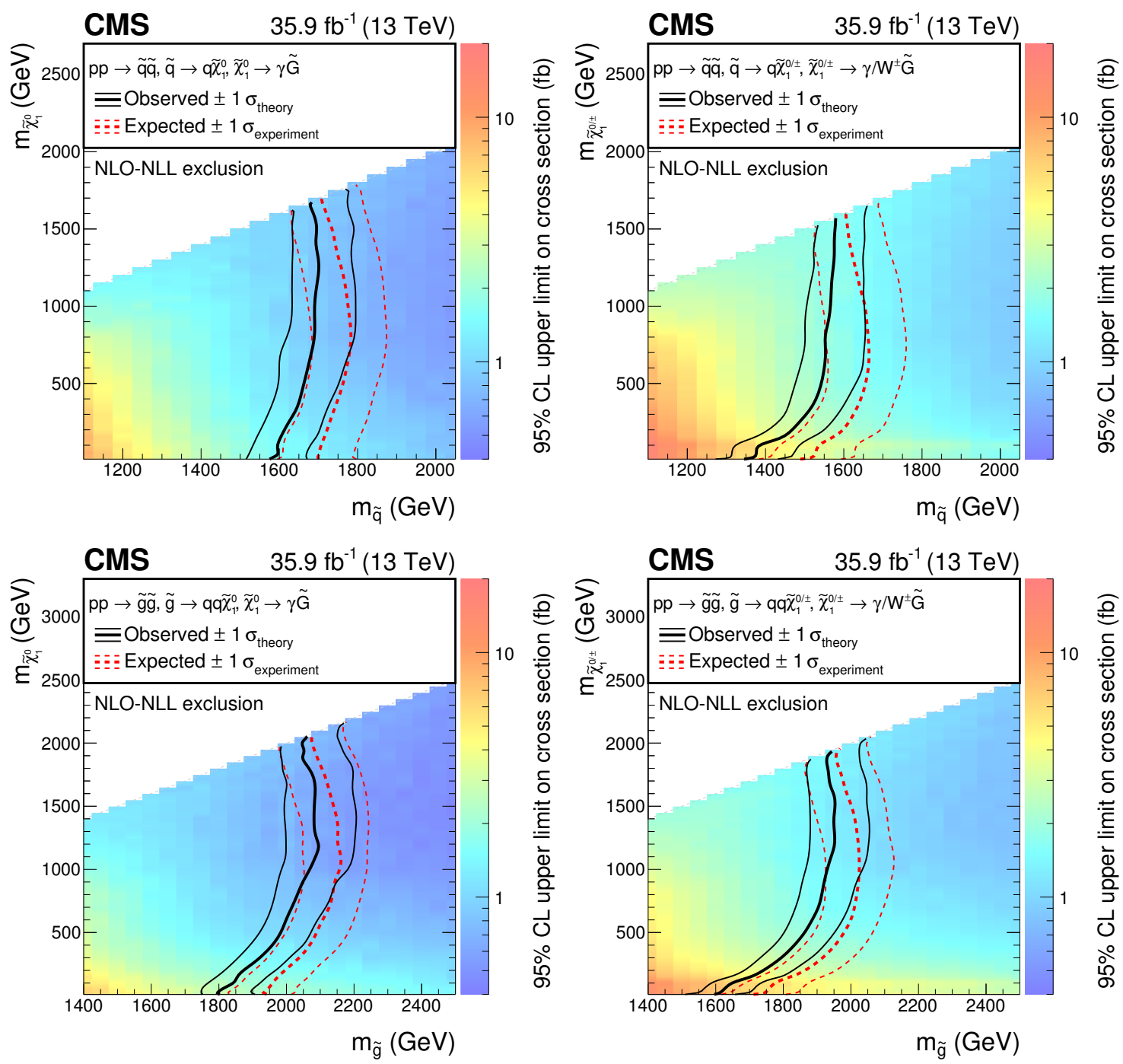

Figure 6. Exclusion limits at 95\% CL for the T6gg (top left), T6Wg (top right), T5gg (bottom left) and $\mathrm{T} 5 \mathrm{Wg}$ (bottom right) models. The solid black curve represents the observed exclusion contour and the uncertainty due to the signal cross section. The red dashed curves represent the expected exclusion contours and the experimental uncertainties.

CEA and CNRS/IN2P3 (France); BMBF, DFG, and HGF (Germany); GSRT (Greece); OTKA and NIH (Hungary); DAE and DST (India); IPM (Iran); SFI (Ireland); INFN (Italy); MSIP and NRF (Republic of Korea); LAS (Lithuania); MOE and UM (Malaysia); BUAP, CINVESTAV, CONACYT, LNS, SEP, and UASLP-FAI (Mexico); MBIE (New Zealand); PAEC (Pakistan); MSHE and NSC (Poland); FCT (Portugal); JINR (Dubna); MON, RosAtom, RAS, RFBR and RAEP (Russia); MESTD (Serbia); SEIDI, CPAN, PCTI and FEDER (Spain); Swiss Funding Agencies (Switzerland); MST (Taipei); ThEPCenter, IPST, STAR, and NSTDA (Thailand); TUBITAK and TAEK (Turkey); NASU and SFFR (Ukraine); STFC (United Kingdom); DOE and NSF (U.S.A.). 
Individuals have received support from the Marie-Curie programme and the European Research Council and Horizon 2020 Grant, contract No. 675440 (European Union); the Leventis Foundation; the A. P. Sloan Foundation; the Alexander von Humboldt Foundation; the Belgian Federal Science Policy Office; the Fonds pour la Formation à la Recherche dans l'Industrie et dans l'Agriculture (FRIA-Belgium); the Agentschap voor Innovatie door Wetenschap en Technologie (IWT-Belgium); the Ministry of Education, Youth and Sports (MEYS) of the Czech Republic; the Council of Science and Industrial Research, India; the HOMING PLUS programme of the Foundation for Polish Science, cofinanced from European Union, Regional Development Fund, the Mobility Plus programme of the Ministry of Science and Higher Education, the National Science Center (Poland), contracts Harmonia 2014/14/M/ST2/00428, Opus 2014/13/B/ST2/02543, 2014/15/B/ST2/03998, and 2015/19/B/ST2/02861, Sonata-bis 2012/07/E/ST2/01406; the National Priorities Research Program by Qatar National Research Fund; the Programa Clarín-COFUND del Principado de Asturias; the Thalis and Aristeia programmes cofinanced by EU-ESF and the Greek NSRF; the Rachadapisek Sompot Fund for Postdoctoral Fellowship, Chulalongkorn University and the Chulalongkorn Academic into Its 2nd Century Project Advancement Project (Thailand); and the Welch Foundation, contract C-1845.

Open Access. This article is distributed under the terms of the Creative Commons Attribution License (CC-BY 4.0), which permits any use, distribution and reproduction in any medium, provided the original author(s) and source are credited.

\section{References}

[1] R. Barbieri and G.F. Giudice, Upper bounds on supersymmetric particle masses, Nucl. Phys. B 306 (1988) 63 [INSPIRE].

[2] P. Ramond, Dual theory for free fermions, Phys. Rev. D 3 (1971) 2415 [InSPIRE].

[3] Yu. A. Gol'fand and E.P. Likhtman, Extension of the algebra of Poincaré group generators and violation of P invariance, JETP Lett. 13 (1971) 323 [INSPIRE].

[4] S. Ferrara and B. Zumino, Supergauge Invariant Yang-Mills Theories, Nucl. Phys. B 79 (1974) 413 [INSPIRE].

[5] J. Wess and B. Zumino, Supergauge transformations in four-dimensions, Nucl. Phys. B 70 (1974) 39 [INSPIRE].

[6] A.H. Chamseddine, R.L. Arnowitt and P. Nath, Locally supersymmetric grand unification, Phys. Rev. Lett. 49 (1982) 970 [InSPIRE].

[7] R. Barbieri, S. Ferrara and C.A. Savoy, Gauge models with spontaneously broken local supersymmetry, Phys. Lett. B 119 (1982) 343 [INSPIRE].

[8] L.J. Hall, J.D. Lykken and S. Weinberg, Supergravity as the messenger of supersymmetry breaking, Phys. Rev. D 27 (1983) 2359 [INSPIRE].

[9] P. Fayet, Mixing between gravitational and weak interactions through the massive gravitino, Phys. Lett. B 70 (1977) 461 [InSPIRE].

[10] H. Baer, M. Brhlik, C.-h. Chen and X. Tata, Signals for the minimal gauge mediated supersymmetry breaking model at the Fermilab Tevatron collider, Phys. Rev. D 55 (1997) 4463 [hep-ph/9610358] [INSPIRE]. 
[11] H. Baer, P.G. Mercadante, X. Tata and Y.-l. Wang, The reach of Tevatron upgrades in gauge mediated supersymmetry breaking models, Phys. Rev. D 60 (1999) 055001 [hep-ph/9903333] [INSPIRE].

[12] S. Dimopoulos, S.D. Thomas and J.D. Wells, Sparticle spectroscopy and electroweak symmetry breaking with gauge mediated supersymmetry breaking, Nucl. Phys. B 488 (1997) 39 [hep-ph/9609434] [INSPIRE].

[13] J.R. Ellis, J.L. Lopez and D.V. Nanopoulos, Analysis of LEP constraints on supersymmetric models with a light gravitino, Phys. Lett. B 394 (1997) 354 [hep-ph/9610470] [INSPIRE].

[14] M. Dine, A.E. Nelson, Y. Nir and Y. Shirman, New tools for low-energy dynamical supersymmetry breaking, Phys. Rev. D 53 (1996) 2658 [hep-ph/9507378] [InSPIRE].

[15] G.F. Giudice and R. Rattazzi, Gauge-mediated supersymmetry breaking, in Perspectives on supersymmetry, World Scientific, Singapore, (1998), p. 355.

[16] P. Grajek, A. Mariotti and D. Redigolo, Phenomenology of general gauge mediation in light of a 125 GeV Higgs, JHEP 07 (2013) 109 [arXiv: 1303.0870] [INSPIRE].

[17] G.R. Farrar and P. Fayet, Phenomenology of the production, decay and detection of new hadronic states associated with supersymmetry, Phys. Lett. B 76 (1978) 575 [INSPIRE].

[18] R. Barbier et al., R-parity violating supersymmetry, Phys. Rept. 420 (2005) 1 [hep-ph/0406039] [INSPIRE].

[19] J.T. Ruderman and D. Shih, General neutralino NLSPs at the early LHC, JHEP 08 (2012) 159 [arXiv: 1103.6083] [INSPIRE].

[20] CMS collaboration, Search for supersymmetry with photons in pp collisions at $\sqrt{s}=8 \mathrm{TeV}$, Phys. Rev. D 92 (2015) 072006 [arXiv: 1507.02898] [INSPIRE].

[21] CMS collaboration, Search for supersymmetry in electroweak production with photons and large missing transverse energy in pp collisions at $\sqrt{s}=8 \mathrm{TeV}$, Phys. Lett. B 759 (2016) 479 [arXiv: 1602.08772] [INSPIRE].

[22] CMS collaboration, Search for supersymmetry in events with photons and missing transverse energy in pp collisions at 13 TeV, Phys. Lett. B 769 (2017) 391 [arXiv:1611.06604] [INSPIRE].

[23] ATLAS collaboration, Search for photonic signatures of gauge-mediated supersymmetry in $8 \mathrm{TeV}$ pp collisions with the ATLAS detector, Phys. Rev. D 92 (2015) 072001 [arXiv: 1507.05493] [INSPIRE].

[24] ATLAS collaboration, Search for supersymmetry in a final state containing two photons and missing transverse momentum in $\sqrt{s}=13 \mathrm{TeV}$ pp collisions at the LHC using the ATLAS detector, Eur. Phys. J. C 76 (2016) 517 [arXiv:1606.09150] [INSPIRE].

[25] CMS collaboration, Jet energy scale and resolution in the CMS experiment in pp collisions at $8 \mathrm{TeV}, 2017$ JINST $12 \mathrm{P} 02014$ [arXiv:1607.03663] [INSPIRE].

[26] CMS collaboration, The CMS Experiment at the CERN LHC, 2008 JINST 3 S08004 [INSPIRE].

[27] CMS collaboration, Particle-flow reconstruction and global event description with the CMS detector, 2017 JINST 12 P10003 [arXiv:1706.04965] [INSPIRE].

[28] M. Cacciari, G.P. Salam and G. Soyez, The anti-kt jet clustering algorithm, JHEP 04 (2008) 063 [arXiv: 0802.1189] [INSPIRE].

[29] M. Cacciari, G.P. Salam and G. Soyez, FastJet user manual, Eur. Phys. J. C 72 (2012) 1896 [arXiv:1111.6097] [INSPIRE]. 
[30] M. Cacciari and G.P. Salam, Pileup subtraction using jet areas, Phys. Lett. B 659 (2008) 119 [arXiv: 0707.1378] [INSPIRE].

[31] J. Alwall et al., The automated computation of tree-level and next-to-leading order differential cross sections and their matching to parton shower simulations, JHEP 07 (2014) 079 [arXiv: 1405.0301] [INSPIRE].

[32] NNPDF collaboration, R.D. Ball et al., Parton distributions for the LHC Run II, JHEP 04 (2015) 040 [arXiv: 1410.8849] [INSPIRE].

[33] T. Sjöstrand, S. Mrenna and P.Z. Skands, A brief introduction to PYTHIA 8.1, Comput. Phys. Commun. 178 (2008) 852 [arXiv:0710.3820] [INSPIRE].

[34] CMS collaboration, Event generator tunes obtained from underlying event and multiparton scattering measurements, Eur. Phys. J. C 76 (2016) 155 [arXiv:1512.00815] [INSPIRE].

[35] C. Borschensky et al., Squark and gluino production cross sections in pp collisions at $\sqrt{s}=13,14,33$ and $100 \mathrm{TeV}$, Eur. Phys. J. C 74 (2014) 3174 [arXiv:1407.5066] [inSPIRE].

[36] LHC New Physics Working Group collaboration, D. Alves, Simplified models for LHC new physics searches, J. Phys. G 39 (2012) 105005 [arXiv:1105.2838] [InSPIRE].

[37] CMS collaboration, Interpretation of searches for supersymmetry with simplified models, Phys. Rev. D 88 (2013) 052017 [arXiv:1301.2175] [InSPIRE].

[38] GEANT4 collaboration, S. Agostinelli et al., GEANT4-a simulation toolkit, Nucl. Instrum. Meth. A 506 (2003) 250 [INSPIRE].

[39] CMS collaboration, S. Abdullin, P. Azzi, F. Beaudette, P. Janot and A. Perrotta, The fast simulation of the CMS detector at LHC, J. Phys. Conf. Ser. 331 (2011) 032049 [InSPIRE].

[40] CMS collaboration, S. Sekmen, Recent developments in CMS fast simulation, PoS (ICHEP2016) 181 [arXiv: 1701.03850] [INSPIRE].

[41] CMS collaboration, The CMS trigger system, 2017 JINST 12 P01020 [arXiv:1609.02366] [INSPIRE].

[42] CMS collaboration, Performance of missing energy reconstruction in $13 \mathrm{TeV}$ pp collision data using the CMS detector, CMS-PAS-JME-16-004 [INSPIRE].

[43] J. Butterworth et al., PDF4LHC recommendations for LHC Run II, J. Phys. G 43 (2016) 023001 [arXiv: 1510.03865] [INSPIRE].

[44] CMS collaboration, CMS luminosity measurements for the 2016 data taking period, CMS-PAS-LUM-17-001 [INSPIRE].

[45] T. Junk, Confidence level computation for combining searches with small statistics, Nucl. Instrum. Meth. A 434 (1999) 435 [hep-ex/9902006] [INSPIRE].

[46] A.L. Read, Presentation of search results: The $C L_{s}$ technique, J. Phys. G 28 (2002) 2693 [INSPIRE].

[47] ATLAS and CMS collaborations, Procedure for the LHC Higgs boson search combination in Summer 2011, CMS-NOTE-2011-005 [INSPIRE].

[48] G. Cowan, K. Cranmer, E. Gross and O. Vitells, Asymptotic formulae for likelihood-based tests of new physics, Eur. Phys. J. C 71 (2011) 1554 [Erratum ibid. C 73 (2013) 2501] [arXiv: 1007.1727] [INSPIRE]. 


\section{The CMS collaboration}

\section{Yerevan Physics Institute, Yerevan, Armenia}

A.M. Sirunyan, A. Tumasyan

\section{Institut für Hochenergiephysik, Wien, Austria}

W. Adam, F. Ambrogi, E. Asilar, T. Bergauer, J. Brandstetter, E. Brondolin, M. Dragicevic, J. Erö, M. Flechl, M. Friedl, R. Frühwirth ${ }^{1}$, V.M. Ghete, J. Grossmann, J. Hrubec, M. Jeitler ${ }^{1}$, A. König, N. Krammer, I. Krätschmer, D. Liko, T. Madlener, I. Mikulec, E. Pree, D. Rabady, N. Rad, H. Rohringer, J. Schieck ${ }^{1}$, R. Schöfbeck, M. Spanring, D. Spitzbart, J. Strauss, W. Waltenberger, J. Wittmann, C.-E. Wulz ${ }^{1}$, M. Zarucki

Institute for Nuclear Problems, Minsk, Belarus

V. Chekhovsky, V. Mossolov, J. Suarez Gonzalez

\section{Universiteit Antwerpen, Antwerpen, Belgium}

E.A. De Wolf, D. Di Croce, X. Janssen, J. Lauwers, H. Van Haevermaet, P. Van Mechelen, N. Van Remortel

\section{Vrije Universiteit Brussel, Brussel, Belgium}

S. Abu Zeid, F. Blekman, J. D’Hondt, I. De Bruyn, J. De Clercq, K. Deroover, G. Flouris, D. Lontkovskyi, S. Lowette, S. Moortgat, L. Moreels, A. Olbrechts, Q. Python, K. Skovpen, S. Tavernier, W. Van Doninck, P. Van Mulders, I. Van Parijs

\section{Université Libre de Bruxelles, Bruxelles, Belgium}

H. Brun, B. Clerbaux, G. De Lentdecker, H. Delannoy, G. Fasanella, L. Favart, R. Goldouzian, A. Grebenyuk, G. Karapostoli, T. Lenzi, J. Luetic, T. Maerschalk, A. Marinov, A. Randle-conde, T. Seva, C. Vander Velde, P. Vanlaer, D. Vannerom, R. Yonamine, F. Zenoni, F. Zhang ${ }^{2}$

\section{Ghent University, Ghent, Belgium}

A. Cimmino, T. Cornelis, D. Dobur, A. Fagot, M. Gul, I. Khvastunov, D. Poyraz, C. Roskas, S. Salva, M. Tytgat, W. Verbeke, N. Zaganidis

\section{Université Catholique de Louvain, Louvain-la-Neuve, Belgium}

H. Bakhshiansohi, O. Bondu, S. Brochet, G. Bruno, A. Caudron, S. De Visscher, C. Delaere, M. Delcourt, B. Francois, A. Giammanco, A. Jafari, M. Komm, G. Krintiras, V. Lemaitre, A. Magitteri, A. Mertens, M. Musich, K. Piotrzkowski, L. Quertenmont, M. Vidal Marono, S. Wertz

\section{Université de Mons, Mons, Belgium}

N. Beliy

\section{Centro Brasileiro de Pesquisas Fisicas, Rio de Janeiro, Brazil}

W.L. Aldá Júnior, F.L. Alves, G.A. Alves, L. Brito, M. Correa Martins Junior, C. Hensel, A. Moraes, M.E. Pol, P. Rebello Teles 
Universidade do Estado do Rio de Janeiro, Rio de Janeiro, Brazil

E. Belchior Batista Das Chagas, W. Carvalho, J. Chinellato ${ }^{3}$, A. Custódio, E.M. Da Costa, G.G. Da Silveira ${ }^{4}$, D. De Jesus Damiao, S. Fonseca De Souza, L.M. Huertas Guativa, H. Malbouisson, M. Melo De Almeida, C. Mora Herrera, L. Mundim, H. Nogima, A. Santoro, A. Sznajder, E.J. Tonelli Manganote ${ }^{3}$, F. Torres Da Silva De Araujo, A. Vilela Pereira Universidade Estadual Paulista $^{a}$, Universidade Federal do ABC ${ }^{b}$, São Paulo, Brazil

S. Ahuja $^{a}$, C.A. Bernardes ${ }^{a}$, T.R. Fernandez Perez Tomei ${ }^{a}$, E.M. Gregores ${ }^{b}$, P.G. Mercadante ${ }^{b}$, S.F. Novaes ${ }^{a}$, Sandra S. Padula ${ }^{a}$, D. Romero Abad ${ }^{b}$, J.C. Ruiz $\operatorname{Vargas}^{a}$

Institute for Nuclear Research and Nuclear Energy of Bulgaria Academy of Sciences

A. Aleksandrov, R. Hadjiiska, P. Iaydjiev, M. Misheva, M. Rodozov, M. Shopova, S. Stoykova, G. Sultanov

University of Sofia, Sofia, Bulgaria

A. Dimitrov, I. Glushkov, L. Litov, B. Pavlov, P. Petkov

Beihang University, Beijing, China

W. Fang ${ }^{5}$, X. Gao ${ }^{5}$

Institute of High Energy Physics, Beijing, China

M. Ahmad, J.G. Bian, G.M. Chen, H.S. Chen, M. Chen, Y. Chen, C.H. Jiang, D. Leggat, H. Liao, Z. Liu, F. Romeo, S.M. Shaheen, A. Spiezia, J. Tao, C. Wang, Z. Wang, E. Yazgan, H. Zhang, J. Zhao

State Key Laboratory of Nuclear Physics and Technology, Peking University, Beijing, China

Y. Ban, G. Chen, Q. Li, S. Liu, Y. Mao, S.J. Qian, D. Wang, Z. Xu

Universidad de Los Andes, Bogota, Colombia

C. Avila, A. Cabrera, L.F. Chaparro Sierra, C. Florez, C.F. González Hernández, J.D. Ruiz Alvarez

University of Split, Faculty of Electrical Engineering, Mechanical Engineering and Naval Architecture, Split, Croatia

B. Courbon, N. Godinovic, D. Lelas, I. Puljak, P.M. Ribeiro Cipriano, T. Sculac

University of Split, Faculty of Science, Split, Croatia

Z. Antunovic, M. Kovac

Institute Rudjer Boskovic, Zagreb, Croatia

V. Brigljevic, D. Ferencek, K. Kadija, B. Mesic, A. Starodumov ${ }^{6}$, T. Susa

University of Cyprus, Nicosia, Cyprus

M.W. Ather, A. Attikis, G. Mavromanolakis, J. Mousa, C. Nicolaou, F. Ptochos, P.A. Razis, H. Rykaczewski

Charles University, Prague, Czech Republic

M. Finger ${ }^{7}$, M. Finger Jr. ${ }^{7}$ 
Universidad San Francisco de Quito, Quito, Ecuador

E. Carrera Jarrin

Academy of Scientific Research and Technology of the Arab Republic of Egypt, Egyptian Network of High Energy Physics, Cairo, Egypt

A. Ellithi Kamel ${ }^{8}$, S. Khalil ${ }^{9}$, A. Mohamed ${ }^{9}$

National Institute of Chemical Physics and Biophysics, Tallinn, Estonia

R.K. Dewanjee, M. Kadastik, L. Perrini, M. Raidal, A. Tiko, C. Veelken

Department of Physics, University of Helsinki, Helsinki, Finland

P. Eerola, J. Pekkanen, M. Voutilainen

Helsinki Institute of Physics, Helsinki, Finland

J. Härkönen, T. Järvinen, V. Karimäki, R. Kinnunen, T. Lampén, K. Lassila-Perini,

S. Lehti, T. Lindén, P. Luukka, E. Tuominen, J. Tuominiemi, E. Tuovinen

Lappeenranta University of Technology, Lappeenranta, Finland

J. Talvitie, T. Tuuva

IRFU, CEA, Université Paris-Saclay, Gif-sur-Yvette, France

M. Besancon, F. Couderc, M. Dejardin, D. Denegri, J.L. Faure, F. Ferri, S. Ganjour, S. Ghosh, A. Givernaud, P. Gras, G. Hamel de Monchenault, P. Jarry, I. Kucher, E. Locci, M. Machet, J. Malcles, G. Negro, J. Rander, A. Rosowsky, M.Ö. Sahin, M. Titov

Laboratoire Leprince-Ringuet, Ecole polytechnique, CNRS/IN2P3, Université Paris-Saclay, Palaiseau, France

A. Abdulsalam, I. Antropov, S. Baffioni, F. Beaudette, P. Busson, L. Cadamuro, C. Charlot, R. Granier de Cassagnac, M. Jo, S. Lisniak, A. Lobanov, J. Martin Blanco, M. Nguyen, C. Ochando, G. Ortona, P. Paganini, P. Pigard, S. Regnard, R. Salerno, J.B. Sauvan, Y. Sirois, A.G. Stahl Leiton, T. Strebler, Y. Yilmaz, A. Zabi

Université de Strasbourg, CNRS, IPHC UMR 7178, F-67000 Strasbourg, France

J.-L. Agram ${ }^{10}$, J. Andrea, D. Bloch, J.-M. Brom, M. Buttignol, E.C. Chabert, N. Chanon, C. Collard, E. Conte ${ }^{10}$, X. Coubez, J.-C. Fontaine ${ }^{10}$, D. Gelé, U. Goerlach, M. Jansová, A.-C. Le Bihan, N. Tonon, P. Van Hove

Centre de Calcul de l'Institut National de Physique Nucleaire et de Physique des Particules, CNRS/IN2P3, Villeurbanne, France

S. Gadrat

Université de Lyon, Université Claude Bernard Lyon 1, CNRS-IN2P3, Institut de Physique Nucléaire de Lyon, Villeurbanne, France

S. Beauceron, C. Bernet, G. Boudoul, R. Chierici, D. Contardo, P. Depasse, H. El Mamouni, J. Fay, L. Finco, S. Gascon, M. Gouzevitch, G. Grenier, B. Ille, F. Lagarde, I.B. Laktineh, M. Lethuillier, L. Mirabito, A.L. Pequegnot, S. Perries, A. Popov ${ }^{11}$, V. Sordini, M. Vander Donckt, S. Viret 
Georgian Technical University, Tbilisi, Georgia

T. Toriashvili ${ }^{12}$

\section{Tbilisi State University, Tbilisi, Georgia}

Z. Tsamalaidze ${ }^{7}$

RWTH Aachen University, I. Physikalisches Institut, Aachen, Germany

C. Autermann, S. Beranek, L. Feld, M.K. Kiesel, K. Klein, M. Lipinski, M. Preuten, C. Schomakers, J. Schulz, T. Verlage

RWTH Aachen University, III. Physikalisches Institut A, Aachen, Germany A. Albert, E. Dietz-Laursonn, D. Duchardt, M. Endres, M. Erdmann, S. Erdweg, T. Esch, R. Fischer, A. Güth, M. Hamer, T. Hebbeker, C. Heidemann, K. Hoepfner, S. Knutzen, M. Merschmeyer, A. Meyer, P. Millet, S. Mukherjee, M. Olschewski, K. Padeken, T. Pook, M. Radziej, H. Reithler, M. Rieger, F. Scheuch, D. Teyssier, S. Thüer

RWTH Aachen University, III. Physikalisches Institut B, Aachen, Germany G. Flügge, B. Kargoll, T. Kress, A. Künsken, J. Lingemann, T. Müller, A. Nehrkorn, A. Nowack, C. Pistone, O. Pooth, A. Stahl ${ }^{13}$

\section{Deutsches Elektronen-Synchrotron, Hamburg, Germany}

M. Aldaya Martin, T. Arndt, C. Asawatangtrakuldee, K. Beernaert, O. Behnke, U. Behrens, A. Bermúdez Martínez, A.A. Bin Anuar, K. Borras ${ }^{14}$, V. Botta, A. Campbell, P. Connor, C. Contreras-Campana, F. Costanza, C. Diez Pardos, G. Eckerlin, D. Eckstein, T. Eichhorn, E. Eren, E. Gallo ${ }^{15}$, J. Garay Garcia, A. Geiser, A. Gizhko, J.M. Grados Luyando, A. Grohsjean, P. Gunnellini, A. Harb, J. Hauk, M. Hempel ${ }^{16}$, H. Jung, A. Kalogeropoulos, M. Kasemann, J. Keaveney, C. Kleinwort, I. Korol, D. Krücker, W. Lange, A. Lelek, T. Lenz, J. Leonard, K. Lipka, W. Lohmann ${ }^{16}$, R. Mankel, I.A. Melzer-Pellmann, A.B. Meyer, G. Mittag, J. Mnich, A. Mussgiller, E. Ntomari, D. Pitzl, R. Placakyte, A. Raspereza, B. Roland, M. Savitskyi, P. Saxena, R. Shevchenko, S. Spannagel, N. Stefaniuk, G.P. Van Onsem, R. Walsh, Y. Wen, K. Wichmann, C. Wissing, O. Zenaiev

\section{University of Hamburg, Hamburg, Germany}

S. Bein, V. Blobel, M. Centis Vignali, A.R. Draeger, T. Dreyer, E. Garutti, D. Gonzalez, J. Haller, A. Hinzmann, M. Hoffmann, A. Karavdina, R. Klanner, R. Kogler, N. Kovalchuk, S. Kurz, T. Lapsien, I. Marchesini, D. Marconi, M. Meyer, M. Niedziela, D. Nowatschin, F. Pantaleo ${ }^{13}$, T. Peiffer, A. Perieanu, C. Scharf, P. Schleper, A. Schmidt, S. Schumann, J. Schwandt, J. Sonneveld, H. Stadie, G. Steinbrück, F.M. Stober, M. Stöver, H. Tholen, D. Troendle, E. Usai, L. Vanelderen, A. Vanhoefer, B. Vormwald

\section{Institut für Experimentelle Kernphysik, Karlsruhe, Germany}

M. Akbiyik, C. Barth, S. Baur, E. Butz, R. Caspart, T. Chwalek, F. Colombo, W. De Boer, A. Dierlamm, B. Freund, R. Friese, M. Giffels, A. Gilbert, D. Haitz, F. Hartmann ${ }^{13}$, S.M. Heindl, U. Husemann, F. Kassel ${ }^{13}$, S. Kudella, H. Mildner, M.U. Mozer, Th. Müller, M. Plagge, G. Quast, K. Rabbertz, M. Schröder, I. Shvetsov, G. Sieber, H.J. Simonis, R. Ulrich, S. Wayand, M. Weber, T. Weiler, S. Williamson, C. Wöhrmann, R. Wolf 
Institute of Nuclear and Particle Physics (INPP), NCSR Demokritos, Aghia Paraskevi, Greece

G. Anagnostou, G. Daskalakis, T. Geralis, V.A. Giakoumopoulou, A. Kyriakis, D. Loukas, I. Topsis-Giotis

National and Kapodistrian University of Athens, Athens, Greece

S. Kesisoglou, A. Panagiotou, N. Saoulidou

University of Ioánnina, Ioánnina, Greece

I. Evangelou, C. Foudas, P. Kokkas, S. Mallios, N. Manthos, I. Papadopoulos, E. Paradas, J. Strologas, F.A. Triantis

MTA-ELTE Lendület CMS Particle and Nuclear Physics Group, Eötvös Loránd University, Budapest, Hungary

M. Csanad, N. Filipovic, G. Pasztor

Wigner Research Centre for Physics, Budapest, Hungary

G. Bencze, C. Hajdu, D. Horvath ${ }^{17}$, Á. Hunyadi, F. Sikler, V. Veszpremi, G. Vesztergombi ${ }^{18}$, A.J. Zsigmond

Institute of Nuclear Research ATOMKI, Debrecen, Hungary

N. Beni, S. Czellar, J. Karancsi ${ }^{19}$, A. Makovec, J. Molnar, Z. Szillasi

Institute of Physics, University of Debrecen, Debrecen, Hungary

M. Bartók ${ }^{18}$, P. Raics, Z.L. Trocsanyi, B. Ujvari

Indian Institute of Science (IISc), Bangalore, India

S. Choudhury, J.R. Komaragiri

National Institute of Science Education and Research, Bhubaneswar, India

S. Bahinipati ${ }^{20}$, S. Bhowmik, P. Mal, K. Mandal, A. Nayak ${ }^{21}$, D.K. Sahoo ${ }^{20}$, N. Sahoo, S.K. Swain

Panjab University, Chandigarh, India

S. Bansal, S.B. Beri, V. Bhatnagar, U. Bhawandeep, R. Chawla, N. Dhingra, A.K. Kalsi,

A. Kaur, M. Kaur, R. Kumar, P. Kumari, A. Mehta, J.B. Singh, G. Walia

University of Delhi, Delhi, India

Ashok Kumar, Aashaq Shah, A. Bhardwaj, S. Chauhan, B.C. Choudhary, R.B. Garg, S. Keshri, A. Kumar, S. Malhotra, M. Naimuddin, K. Ranjan, R. Sharma, V. Sharma

Saha Institute of Nuclear Physics, HBNI, Kolkata, India

R. Bhardwaj, R. Bhattacharya, S. Bhattacharya, S. Dey, S. Dutt, S. Dutta, S. Ghosh, N. Majumdar, A. Modak, K. Mondal, S. Mukhopadhyay, S. Nandan, A. Purohit, A. Roy, D. Roy, S. Roy Chowdhury, S. Sarkar, M. Sharan, S. Thakur

Indian Institute of Technology Madras, Madras, India P.K. Behera 


\section{Bhabha Atomic Research Centre, Mumbai, India}

R. Chudasama, D. Dutta, V. Jha, V. Kumar, A.K. Mohanty ${ }^{13}$, P.K. Netrakanti, L.M. Pant, P. Shukla, A. Topkar

Tata Institute of Fundamental Research-A, Mumbai, India

T. Aziz, S. Dugad, B. Mahakud, S. Mitra, G.B. Mohanty, B. Parida, N. Sur, B. Sutar

\section{Tata Institute of Fundamental Research-B, Mumbai, India}

S. Banerjee, S. Bhattacharya, S. Chatterjee, P. Das, M. Guchait, Sa. Jain, S. Kumar, M. Maity ${ }^{22}$, G. Majumder, K. Mazumdar, T. Sarkar ${ }^{22}$, N. Wickramage ${ }^{23}$

Indian Institute of Science Education and Research (IISER), Pune, India

S. Chauhan, S. Dube, V. Hegde, A. Kapoor, K. Kothekar, S. Pandey, A. Rane, S. Sharma

Institute for Research in Fundamental Sciences (IPM), Tehran, Iran

S. Chenarani ${ }^{24}$, E. Eskandari Tadavani, S.M. Etesami ${ }^{24}$, M. Khakzad, M. Mohammadi Najafabadi, M. Naseri, S. Paktinat Mehdiabadi ${ }^{25}$, F. Rezaei Hosseinabadi, B. Safarzadeh ${ }^{26}$, M. Zeinali

\section{University College Dublin, Dublin, Ireland}

M. Felcini, M. Grunewald

INFN Sezione di Bari ${ }^{a}$, Università di Bari ${ }^{b}$, Politecnico di Bari ${ }^{c}$, Bari, Italy

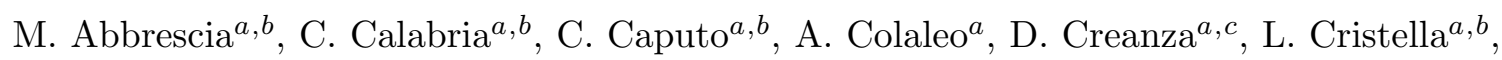
N. De Filippis ${ }^{a, c}$, M. De Palma ${ }^{a, b}$, F. Errico ${ }^{a, b}$, L. Fiore $^{a}$, G. Iaselli ${ }^{a, c}$, S. Lezki ${ }^{a, b}$, G. Maggi ${ }^{a, c}$, M. Maggi ${ }^{a}$, G. Miniello ${ }^{a, b}, \mathrm{~S} . \mathrm{My}^{a}, b, \mathrm{~S} . \mathrm{Nuzzo}^{a, b}$, A. Pompili $^{a, b}$, G. Pugliese $^{a, c}$, R. Radogna ${ }^{a, b}$, A. Ranieri ${ }^{a}$, G. Selvaggi ${ }^{a}, b$ A. Sharma ${ }^{a}$, L. Silvestris ${ }^{a, 13}$, R. Venditti $^{a}$, P. Verwilligen ${ }^{a}$

INFN Sezione di Bologna ${ }^{a}$, Università di Bologna ${ }^{b}$, Bologna, Italy

G. Abbiendi ${ }^{a}$, C. Battilana ${ }^{a, b}$, D. Bonacorsi ${ }^{a}, b$, S. Braibant-Giacomelli ${ }^{a, b}$, R. Campanini ${ }^{a}, b$, P. Capiluppi ${ }^{a}, b$, A. Castro ${ }^{a}, b$, F.R. Cavallo ${ }^{a}$, S.S. Chhibra ${ }^{a}$, G. Codispoti ${ }^{a, b}$, M. Cuffiani $^{a}, b$, G.M. Dallavalle ${ }^{a}$, F. Fabbri ${ }^{a}$, A. Fanfani ${ }^{a}, b$, D. Fasanella ${ }^{a, b}$, P. Giacomelli ${ }^{a}$, C. Grandi ${ }^{a}$, L. Guiducci ${ }^{a}, b$, S. Marcellini ${ }^{a}$, G. Masetti ${ }^{a}$, A. Montanari ${ }^{a}$, F.L. Navarria ${ }^{a}, b$, A. Perrotta $^{a}$, A.M. Rossi ${ }^{a, b}$, T. Rovelli ${ }^{a, b}$, G.P. Siroli ${ }^{a, b}$, N. Tosi ${ }^{a}$

INFN Sezione di Catania ${ }^{a}$, Università di Catania ${ }^{b}$, Catania, Italy

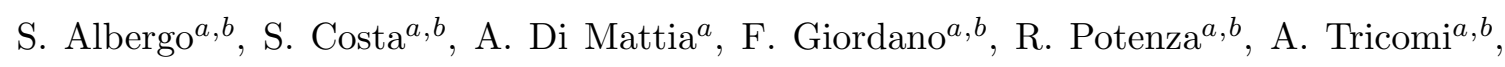
C. Tuve $\mathrm{e}^{a, b}$

INFN Sezione di Firenze ${ }^{a}$, Università di Firenze ${ }^{b}$, Firenze, Italy

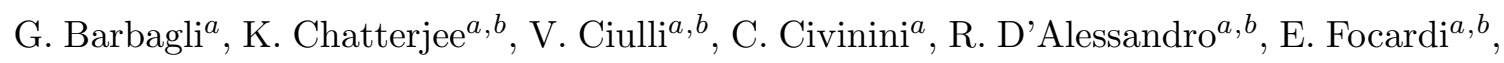

P. Lenzi ${ }^{a, b}$, M. Meschini ${ }^{a}$, S. Paoletti ${ }^{a}$, L. Russo ${ }^{a, 27}$, G. Sguazzoni ${ }^{a}$, D. Strom $^{a}$, L. Viliani ${ }^{a, b, 13}$

INFN Laboratori Nazionali di Frascati, Frascati, Italy

L. Benussi, S. Bianco, F. Fabbri, D. Piccolo, F. Primavera ${ }^{13}$ 
INFN Sezione di Genova ${ }^{a}$, Università di Genova ${ }^{b}$, Genova, Italy V. Calvelli ${ }^{a, b}$, F. Ferro ${ }^{a}$, E. Robutti $^{a}$, S. Tosi $^{a, b}$

INFN Sezione di Milano-Bicocca ${ }^{a}$, Università di Milano-Bicocca ${ }^{b}$, Milano, Italy

L. Brianza ${ }^{a, b}$, F. Brivio ${ }^{a, b}$, V. Ciriolo ${ }^{a, b}$, M.E. Dinardo ${ }^{a, b}$, S. Fiorendi ${ }^{a, b}$, S. Gennai $^{a}$,

A. Ghezzi ${ }^{a, b}$, P. Govoni ${ }^{a, b}$, M. Malberti ${ }^{a}, b$, S. Malvezzi ${ }^{a}$, R.A. Manzoni ${ }^{a}, b$, D. Menasce ${ }^{a}$,

L. Moroni ${ }^{a}$, M. Paganoni ${ }^{a, b}$, K. Pauwels ${ }^{a, b}$, D. Pedrini ${ }^{a}$, S. Pigazzini ${ }^{a, b, 28}$, S. Ragazzi ${ }^{a, b}$,

T. Tabarelli de Fatis ${ }^{a, b}$

INFN Sezione di Napoli ${ }^{a}$, Università di Napoli 'Federico II' ${ }^{b}$, Napoli, Italy, Università della Basilicata ${ }^{c}$, Potenza, Italy, Università G. Marconi ${ }^{d}$, Roma, Italy

S. Buontempo ${ }^{a}$, N. Cavallo ${ }^{a, c}$, S. Di Guida ${ }^{a, d, 13}$, M. Esposito $^{a, b}$, F. Fabozzi $^{a, c}$, F. Fienga $^{a, b}$,

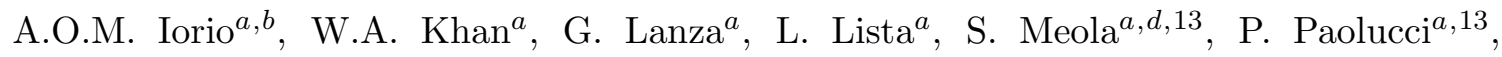
C. Sciacca ${ }^{a, b}$, F. Thyssen ${ }^{a}$

INFN Sezione di Padova ${ }^{a}$, Università di Padova ${ }^{b}$, Padova, Italy, Università di Trento ${ }^{c}$, Trento, Italy

P. Azzi ${ }^{a, 13}$, N. Bacchetta ${ }^{a}$, L. Benato ${ }^{a, b}$, A. Boletti ${ }^{a, b}$, P. Checchia $^{a}$, P. De Castro

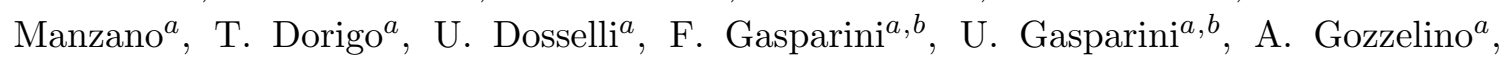
S. Lacaprara ${ }^{a}$, M. Margoni ${ }^{a, b}$, A.T. Meneguzzo ${ }^{a, b}$, D. Pantano ${ }^{a}$, M. Passaseo ${ }^{a}$,

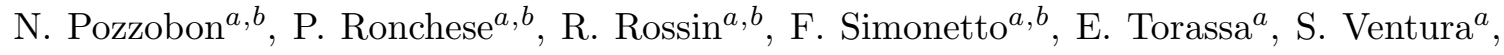
M. Zanetti ${ }^{a, b}$, P. Zotto ${ }^{a, b}$, G. Zumerle ${ }^{a, b}$

INFN Sezione di Pavia ${ }^{a}$, Università di Pavia ${ }^{b}$, Pavia, Italy

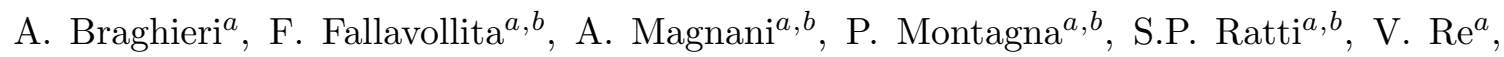
M. Ressegotti, C. Riccardi ${ }^{a, b}$, P. Salvini ${ }^{a}$, I. Vai ${ }^{a, b}$, P. Vitulo ${ }^{a, b}$

INFN Sezione di Perugia ${ }^{a}$, Università di Perugia ${ }^{b}$, Perugia, Italy

L. Alunni Solestizi ${ }^{a, b}$, M. Biasini ${ }^{a, b}$, G.M. Bilei ${ }^{a}$, C. Cecchi ${ }^{a, b}$, D. Ciangottini ${ }^{a, b}$, L. Fanò $^{a, b}$, P. Lariccia ${ }^{a, b}$, R. Leonardi ${ }^{a, b}$, E. Manoni $^{a}$, G. Mantovani ${ }^{a, b}$, V. Mariani $^{a}, b$, M. Menichelli $^{a}$, A. $\operatorname{Rossi}^{a, b}$, A. Santocchia ${ }^{a, b}$, D. Spiga ${ }^{a}$

INFN Sezione di Pisa ${ }^{a}$, Università di Pisa ${ }^{b}$, Scuola Normale Superiore di Pisa ${ }^{c}$, Pisa, Italy

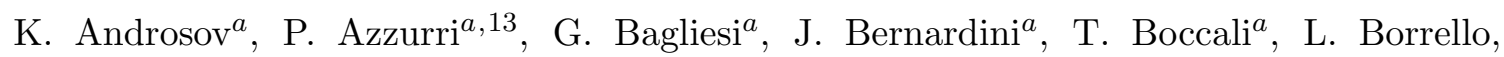

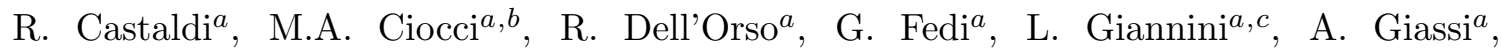
M.T. Grippo ${ }^{a, 27}$, F. Ligabue ${ }^{a, c}$, T. Lomtadze ${ }^{a}$, E. Manca ${ }^{a, c}$, G. Mandorli ${ }^{a, c}$, L. Martini $^{a}, b$, A. Messineo ${ }^{a, b}$, F. Palla ${ }^{a}$, A. Rizzi ${ }^{a, b}$, A. Savoy-Navarro ${ }^{a, 29}$, P. Spagnolo ${ }^{a}$, R. Tenchini ${ }^{a}$, G. Tonelli ${ }^{a, b}$, A. Venturi ${ }^{a}$, P.G. Verdini ${ }^{a}$

INFN Sezione di Roma ${ }^{a}$, Sapienza Università di Roma ${ }^{b}$, Rome, Italy

L. Barone ${ }^{a, b}$, F. Cavallari ${ }^{a}$, M. Cipriani ${ }^{a, b}$, D. Del Re ${ }^{a, b, 13}$, M. Diemoz $^{a}$, S. Gelli ${ }^{a, b}$, E. Longo ${ }^{a, b}$, F. Margaroli ${ }^{a, b}$, B. Marzocchi ${ }^{a, b}$, P. Meridiani ${ }^{a}$, G. Organtini ${ }^{a, b}$,

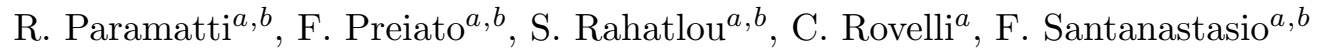


INFN Sezione di Torino ${ }^{a}$, Università di Torino ${ }^{b}$, Torino, Italy, Università del Piemonte Orientale ${ }^{c}$, Novara, Italy

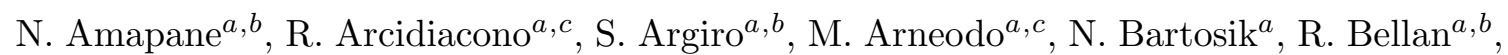
C. Biino ${ }^{a}$, N. Cartiglia ${ }^{a}$, F. Cenna ${ }^{a, b}$, M. Costa ${ }^{a, b}$, R. Covarelli ${ }^{a, b}$, A. Degano ${ }^{a, b}$, N. Demaria ${ }^{a}$, B. Kiani ${ }^{a, b}$, C. Mariotti ${ }^{a}$, S. Maselli ${ }^{a}$, E. Migliore ${ }^{a, b}$, V. Monaco ${ }^{a, b}$,

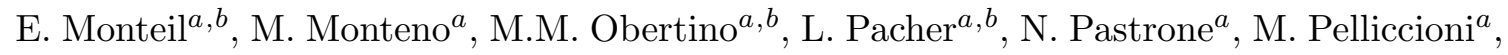
G.L. Pinna Angioni ${ }^{a, b}$, F. Ravera ${ }^{a, b}$, A. Romero ${ }^{a, b}$, M. Ruspa ${ }^{a, c}$, R. Sacchi ${ }^{a, b}$, K. Shchelina ${ }^{a, b}$, V. Sola ${ }^{a}$, A. Solano ${ }^{a, b}$, A. Staiano ${ }^{a}$, P. Traczyk ${ }^{a, b}$

INFN Sezione di Trieste ${ }^{a}$, Università di Trieste ${ }^{b}$, Trieste, Italy

S. Belforte ${ }^{a}$, M. Casarsa ${ }^{a}$, F. Cossutti ${ }^{a}$, G. Della Ricca ${ }^{a}, b$, A. Zanetti ${ }^{a}$

Kyungpook National University, Daegu, Korea

D.H. Kim, G.N. Kim, M.S. Kim, J. Lee, S. Lee, S.W. Lee, C.S. Moon, Y.D. Oh, S. Sekmen, D.C. Son, Y.C. Yang

Chonbuk National University, Jeonju, Korea

A. Lee

Chonnam National University, Institute for Universe and Elementary Particles, Kwangju, Korea

H. Kim, D.H. Moon, G. Oh

Hanyang University, Seoul, Korea

J.A. Brochero Cifuentes, J. Goh, T.J. Kim

Korea University, Seoul, Korea

S. Cho, S. Choi, Y. Go, D. Gyun, S. Ha, B. Hong, Y. Jo, Y. Kim, K. Lee, K.S. Lee, S. Lee, J. Lim, S.K. Park, Y. Roh

Seoul National University, Seoul, Korea

J. Almond, J. Kim, J.S. Kim, H. Lee, K. Lee, K. Nam, S.B. Oh, B.C. Radburn-Smith, S.h. Seo, U.K. Yang, H.D. Yoo, G.B. Yu

University of Seoul, Seoul, Korea

M. Choi, H. Kim, J.H. Kim, J.S.H. Lee, I.C. Park, G. Ryu

Sungkyunkwan University, Suwon, Korea

Y. Choi, C. Hwang, J. Lee, I. Yu

Vilnius University, Vilnius, Lithuania

V. Dudenas, A. Juodagalvis, J. Vaitkus

National Centre for Particle Physics, Universiti Malaya, Kuala Lumpur, Malaysia

I. Ahmed, Z.A. Ibrahim, M.A.B. Md $\mathrm{Ali}^{30}$, F. Mohamad Idris ${ }^{31}$, W.A.T. Wan Abdullah, M.N. Yusli, Z. Zolkapli 
Centro de Investigacion y de Estudios Avanzados del IPN, Mexico City, Mexico H. Castilla-Valdez, E. De La Cruz-Burelo, I. Heredia-De La Cruz ${ }^{32}$, R. Lopez-Fernandez, J. Mejia Guisao, A. Sanchez-Hernandez

Universidad Iberoamericana, Mexico City, Mexico

S. Carrillo Moreno, C. Oropeza Barrera, F. Vazquez Valencia

Benemerita Universidad Autonoma de Puebla, Puebla, Mexico

I. Pedraza, H.A. Salazar Ibarguen, C. Uribe Estrada

Universidad Autónoma de San Luis Potosí, San Luis Potosí, Mexico

A. Morelos Pineda

University of Auckland, Auckland, New Zealand

D. Krofcheck

University of Canterbury, Christchurch, New Zealand

P.H. Butler

National Centre for Physics, Quaid-I-Azam University, Islamabad, Pakistan

A. Ahmad, M. Ahmad, Q. Hassan, H.R. Hoorani, A. Saddique, M.A. Shah, M. Shoaib, M. Waqas

National Centre for Nuclear Research, Swierk, Poland

H. Bialkowska, M. Bluj, B. Boimska, T. Frueboes, M. Górski, M. Kazana, K. Nawrocki,

K. Romanowska-Rybinska, M. Szleper, P. Zalewski

Institute of Experimental Physics, Faculty of Physics, University of Warsaw, Warsaw, Poland

K. Bunkowski, A. Byszuk ${ }^{33}$, K. Doroba, A. Kalinowski, M. Konecki, J. Krolikowski, M. Misiura, M. Olszewski, A. Pyskir, M. Walczak

Laboratório de Instrumentação e Física Experimental de Partículas, Lisboa, Portugal

P. Bargassa, C. Beirão Da Cruz E Silva, B. Calpas, A. Di Francesco, P. Faccioli, M. Gallinaro, J. Hollar, N. Leonardo, L. Lloret Iglesias, M.V. Nemallapudi, J. Seixas, O. Toldaiev, D. Vadruccio, J. Varela

Joint Institute for Nuclear Research, Dubna, Russia

S. Afanasiev, P. Bunin, M. Gavrilenko, I. Golutvin, I. Gorbunov, A. Kamenev, V. Karjavin, A. Lanev, A. Malakhov, V. Matveev ${ }^{34,35}$, V. Palichik, V. Perelygin, S. Shmatov, S. Shulha, N. Skatchkov, V. Smirnov, N. Voytishin, A. Zarubin

Petersburg Nuclear Physics Institute, Gatchina (St. Petersburg), Russia

Y. Ivanov, V. Kim ${ }^{36}$, E. Kuznetsova ${ }^{37}$, P. Levchenko, V. Murzin, V. Oreshkin, I. Smirnov,

V. Sulimov, L. Uvarov, S. Vavilov, A. Vorobyev

Institute for Nuclear Research, Moscow, Russia

Yu. Andreev, A. Dermenev, S. Gninenko, N. Golubev, A. Karneyeu, M. Kirsanov, N. Krasnikov, A. Pashenkov, D. Tlisov, A. Toropin 
Institute for Theoretical and Experimental Physics, Moscow, Russia

V. Epshteyn, V. Gavrilov, N. Lychkovskaya, V. Popov, I. Pozdnyakov, G. Safronov, A. Spiridonov, A. Stepennov, M. Toms, E. Vlasov, A. Zhokin

Moscow Institute of Physics and Technology, Moscow, Russia

T. Aushev, A. Bylinkin ${ }^{35}$

National Research Nuclear University 'Moscow Engineering Physics Institute' (MEPhI), Moscow, Russia

R. Chistov ${ }^{38}$, M. Danilov ${ }^{38}$, P. Parygin, D. Philippov, S. Polikarpov, E. Tarkovskii, E. Zhemchugov

P.N. Lebedev Physical Institute, Moscow, Russia

V. Andreev, M. Azarkin ${ }^{35}$, I. Dremin ${ }^{35}$, M. Kirakosyan ${ }^{35}$, A. Terkulov

Skobeltsyn Institute of Nuclear Physics, Lomonosov Moscow State University, Moscow, Russia

A. Baskakov, A. Belyaev, E. Boos, M. Dubinin ${ }^{39}$, L. Dudko, A. Ershov, A. Gribushin, V. Klyukhin, O. Kodolova, I. Lokhtin, I. Miagkov, S. Obraztsov, S. Petrushanko, V. Savrin, A. Snigirev

Novosibirsk State University (NSU), Novosibirsk, Russia V. Blinov ${ }^{40}$, Y.Skovpen $^{40}$, D. Shtol ${ }^{40}$

State Research Center of Russian Federation, Institute for High Energy Physics, Protvino, Russia

I. Azhgirey, I. Bayshev, S. Bitioukov, D. Elumakhov, V. Kachanov, A. Kalinin, D. Konstantinov, V. Krychkine, V. Petrov, R. Ryutin, A. Sobol, S. Troshin, N. Tyurin, A. Uzunian,

A. Volkov

University of Belgrade, Faculty of Physics and Vinca Institute of Nuclear Sciences, Belgrade, Serbia

P. Adzic ${ }^{41}$, P. Cirkovic, D. Devetak, M. Dordevic, J. Milosevic, V. Rekovic

Centro de Investigaciones Energéticas Medioambientales y Tecnológicas (CIEMAT), Madrid, Spain

J. Alcaraz Maestre, M. Barrio Luna, M. Cerrada, N. Colino, B. De La Cruz, A. Delgado Peris, A. Escalante Del Valle, C. Fernandez Bedoya, J.P. Fernández Ramos, J. Flix, M.C. Fouz, P. Garcia-Abia, O. Gonzalez Lopez, S. Goy Lopez, J.M. Hernandez, M.I. Josa, A. Pérez-Calero Yzquierdo, J. Puerta Pelayo, A. Quintario Olmeda, I. Redondo, L. Romero, M.S. Soares, A. Álvarez Fernández

Universidad Autónoma de Madrid, Madrid, Spain

J.F. de Trocóniz, M. Missiroli, D. Moran

Universidad de Oviedo, Oviedo, Spain

J. Cuevas, C. Erice, J. Fernandez Menendez, I. Gonzalez Caballero, J.R. González Fernández, E. Palencia Cortezon, S. Sanchez Cruz, I. Suárez Andrés, P. Vischia, J.M. Vizan Garcia 
Instituto de Física de Cantabria (IFCA), CSIC-Universidad de Cantabria, Santander, Spain

I.J. Cabrillo, A. Calderon, B. Chazin Quero, E. Curras, M. Fernandez, J. Garcia-Ferrero, G. Gomez, A. Lopez Virto, J. Marco, C. Martinez Rivero, P. Martinez Ruiz del Arbol, F. Matorras, J. Piedra Gomez, T. Rodrigo, A. Ruiz-Jimeno, L. Scodellaro, N. Trevisani, I. Vila, R. Vilar Cortabitarte

\section{CERN, European Organization for Nuclear Research, Geneva, Switzerland}

D. Abbaneo, E. Auffray, P. Baillon, A.H. Ball, D. Barney, M. Bianco, P. Bloch, A. Bocci, C. Botta, T. Camporesi, R. Castello, M. Cepeda, G. Cerminara, E. Chapon, Y. Chen, D. d'Enterria, A. Dabrowski, V. Daponte, A. David, M. De Gruttola, A. De Roeck, E. Di Marco $^{42}$, M. Dobson, B. Dorney, T. du Pree, M. Dünser, N. Dupont, A. Elliott-Peisert, P. Everaerts, G. Franzoni, J. Fulcher, W. Funk, D. Gigi, K. Gill, F. Glege, D. Gulhan, S. Gundacker, M. Guthoff, P. Harris, J. Hegeman, V. Innocente, P. Janot, O. Karacheban ${ }^{16}$, J. Kieseler, H. Kirschenmann, V. Knünz, A. Kornmayer ${ }^{13}$, M.J. Kortelainen, C. Lange, P. Lecoq, C. Lourenço, M.T. Lucchini, L. Malgeri, M. Mannelli, A. Martelli, F. Meijers, J.A. Merlin, S. Mersi, E. Meschi, P. Milenovic ${ }^{43}$, F. Moortgat, M. Mulders, H. Neugebauer, S. Orfanelli, L. Orsini, L. Pape, E. Perez, M. Peruzzi, A. Petrilli, G. Petrucciani, A. Pfeiffer, M. Pierini, A. Racz, T. Reis, G. Rolandi ${ }^{44}$, M. Rovere, H. Sakulin, C. Schäfer, C. Schwick, M. Seidel, M. Selvaggi, A. Sharma, P. Silva, P. Sphicas ${ }^{45}$, J. Steggemann, M. Stoye, M. Tosi, D. Treille, A. Triossi, A. Tsirou, V. Veckalns ${ }^{46}$, G.I. Veres ${ }^{18}$, M. Verweij, N. Wardle, W.D. Zeuner

\section{Paul Scherrer Institut, Villigen, Switzerland}

W. Bertl ${ }^{\dagger}$, L. Caminada ${ }^{47}$, K. Deiters, W. Erdmann, R. Horisberger, Q. Ingram, H.C. Kaestli, D. Kotlinski, U. Langenegger, T. Rohe, S.A. Wiederkehr

Institute for Particle Physics, ETH Zurich, Zurich, Switzerland

F. Bachmair, L. Bäni, P. Berger, L. Bianchini, B. Casal, G. Dissertori, M. Dittmar, M. Donegà, C. Grab, C. Heidegger, D. Hits, J. Hoss, G. Kasieczka, T. Klijnsma, W. Lustermann, B. Mangano, M. Marionneau, M.T. Meinhard, D. Meister, F. Micheli, P. Musella, F. Nessi-Tedaldi, F. Pandolfi, J. Pata, F. Pauss, G. Perrin, L. Perrozzi, M. Quittnat, M. Schönenberger, L. Shchutska, V.R. Tavolaro, K. Theofilatos, M.L. Vesterbacka Olsson, R. Wallny, A. Zagozdzinska ${ }^{33}$, D.H. Zhu

\section{Universität Zürich, Zurich, Switzerland}

T.K. Aarrestad, C. Amsler ${ }^{48}$, M.F. Canelli, A. De Cosa, S. Donato, C. Galloni, T. Hreus, B. Kilminster, J. Ngadiuba, D. Pinna, G. Rauco, P. Robmann, D. Salerno, C. Seitz, A. Zucchetta

\section{National Central University, Chung-Li, Taiwan}

V. Candelise, T.H. Doan, Sh. Jain, R. Khurana, C.M. Kuo, W. Lin, A. Pozdnyakov, S.S. Yu

\section{National Taiwan University (NTU), Taipei, Taiwan}

Arun Kumar, P. Chang, Y. Chao, K.F. Chen, P.H. Chen, F. Fiori, W.-S. Hou, Y. Hsiung, Y.F. Liu, R.-S. Lu, M. Miñano Moya, E. Paganis, A. Psallidas, J.f. Tsai 
Chulalongkorn University, Faculty of Science, Department of Physics, Bangkok, Thailand

B. Asavapibhop, K. Kovitanggoon, G. Singh, N. Srimanobhas

Cukurova University, Physics Department, Science and Art Faculty, Adana, Turkey

A. Adiguzel ${ }^{49}$, M.N. Bakirci ${ }^{50}$, F. Boran, S. Damarseckin, Z.S. Demiroglu, C. Dozen, E. Eskut, S. Girgis, G. Gokbulut, Y. Guler, I. $\mathrm{Hos}^{51}$, E.E. Kangal ${ }^{52}$, O. Kara, U. Kiminsu, M. Oglakci, G. Onengut ${ }^{53}$, K. Ozdemir ${ }^{54}$, S. Ozturk ${ }^{50}$, A. Polatoz, D. Sunar Cerci ${ }^{55}$, S. Turkcapar, I.S. Zorbakir, C. Zorbilmez

Middle East Technical University, Physics Department, Ankara, Turkey

B. Bilin, G. Karapinar ${ }^{56}$, K. Ocalan ${ }^{57}$, M. Yalvac, M. Zeyrek

Bogazici University, Istanbul, Turkey

E. Gülmez, M. Kaya ${ }^{58}$, O. Kaya ${ }^{59}$, S. Tekten, E.A. Yetkin ${ }^{60}$

Istanbul Technical University, Istanbul, Turkey

M.N. Agaras, S. Atay, A. Cakir, K. Cankocak

Institute for Scintillation Materials of National Academy of Science of Ukraine, Kharkov, Ukraine

B. Grynyov

National Scientific Center, Kharkov Institute of Physics and Technology, Kharkov, Ukraine

L. Levchuk, P. Sorokin

University of Bristol, Bristol, United Kingdom

R. Aggleton, F. Ball, L. Beck, J.J. Brooke, D. Burns, E. Clement, D. Cussans, O. Davignon, H. Flacher, J. Goldstein, M. Grimes, G.P. Heath, H.F. Heath, J. Jacob, L. Kreczko, C. Lucas, D.M. Newbold ${ }^{61}$, S. Paramesvaran, A. Poll, T. Sakuma, S. Seif El Nasr-storey, D. Smith, V.J. Smith

Rutherford Appleton Laboratory, Didcot, United Kingdom

K.W. Bell, A. Belyaev ${ }^{62}$, C. Brew, R.M. Brown, L. Calligaris, D. Cieri, D.J.A. Cockerill, J.A. Coughlan, K. Harder, S. Harper, E. Olaiya, D. Petyt, C.H. Shepherd-Themistocleous, A. Thea, I.R. Tomalin, T. Williams

\section{Imperial College, London, United Kingdom}

R. Bainbridge, S. Breeze, O. Buchmuller, A. Bundock, S. Casasso, M. Citron, D. Colling, L. Corpe, P. Dauncey, G. Davies, A. De Wit, M. Della Negra, R. Di Maria, A. Elwood, Y. Haddad, G. Hall, G. Iles, T. James, R. Lane, C. Laner, L. Lyons, A.-M. Magnan, S. Malik, L. Mastrolorenzo, T. Matsushita, J. Nash, A. Nikitenko ${ }^{6}$, V. Palladino, M. Pesaresi, D.M. Raymond, A. Richards, A. Rose, E. Scott, C. Seez, A. Shtipliyski, S. Summers, A. Tapper, K. Uchida, M. Vazquez Acosta ${ }^{63}$, T. Virdee ${ }^{13}$, D. Winterbottom, J. Wright, S.C. Zenz 
Brunel University, Uxbridge, United Kingdom

J.E. Cole, P.R. Hobson, A. Khan, P. Kyberd, I.D. Reid, P. Symonds, L. Teodorescu, M. Turner

Baylor University, Waco, U.S.A.

A. Borzou, K. Call, J. Dittmann, K. Hatakeyama, H. Liu, N. Pastika, C. Smith

Catholic University of America, Washington, U.S.A.

R. Bartek, A. Dominguez

The University of Alabama, Tuscaloosa, U.S.A.

A. Buccilli, S.I. Cooper, C. Henderson, P. Rumerio, C. West

Boston University, Boston, U.S.A.

D. Arcaro, A. Avetisyan, T. Bose, D. Gastler, D. Rankin, C. Richardson, J. Rohlf, L. Sulak, D. Zou

\section{Brown University, Providence, U.S.A.}

G. Benelli, D. Cutts, A. Garabedian, J. Hakala, U. Heintz, J.M. Hogan, K.H.M. Kwok, E. Laird, G. Landsberg, Z. Mao, M. Narain, S. Piperov, S. Sagir, R. Syarif

University of California, Davis, Davis, U.S.A.

R. Band, C. Brainerd, D. Burns, M. Calderon De La Barca Sanchez, M. Chertok, J. Conway, R. Conway, P.T. Cox, R. Erbacher, C. Flores, G. Funk, M. Gardner, W. Ko, R. Lander, C. Mclean, M. Mulhearn, D. Pellett, J. Pilot, S. Shalhout, M. Shi, J. Smith, M. Squires, D. Stolp, K. Tos, M. Tripathi, Z. Wang

University of California, Los Angeles, U.S.A.

M. Bachtis, C. Bravo, R. Cousins, A. Dasgupta, A. Florent, J. Hauser, M. Ignatenko, N. Mccoll, D. Saltzberg, C. Schnaible, V. Valuev

University of California, Riverside, Riverside, U.S.A.

E. Bouvier, K. Burt, R. Clare, J. Ellison, J.W. Gary, S.M.A. Ghiasi Shirazi, G. Hanson, J. Heilman, P. Jandir, E. Kennedy, F. Lacroix, O.R. Long, M. Olmedo Negrete, M.I. Paneva, A. Shrinivas, W. Si, L. Wang, H. Wei, S. Wimpenny, B. R. Yates

University of California, San Diego, La Jolla, U.S.A.

J.G. Branson, S. Cittolin, M. Derdzinski, B. Hashemi, A. Holzner, D. Klein, G. Kole, V. Krutelyov, J. Letts, I. Macneill, M. Masciovecchio, D. Olivito, S. Padhi, M. Pieri, M. Sani, V. Sharma, S. Simon, M. Tadel, A. Vartak, S. Wasserbaech ${ }^{64}$, J. Wood, F. Würthwein, A. Yagil, G. Zevi Della Porta

University of California, Santa Barbara - Department of Physics, Santa Barbara, U.S.A.

N. Amin, R. Bhandari, J. Bradmiller-Feld, C. Campagnari, A. Dishaw, V. Dutta, M. Franco Sevilla, C. George, F. Golf, L. Gouskos, J. Gran, R. Heller, J. Incandela, S.D. Mullin, A. Ovcharova, H. Qu, J. Richman, D. Stuart, I. Suarez, J. Yoo 
California Institute of Technology, Pasadena, U.S.A.

D. Anderson, J. Bendavid, A. Bornheim, J.M. Lawhorn, H.B. Newman, T. Nguyen, C. Pena, M. Spiropulu, J.R. Vlimant, S. Xie, Z. Zhang, R.Y. Zhu

Carnegie Mellon University, Pittsburgh, U.S.A.

M.B. Andrews, T. Ferguson, T. Mudholkar, M. Paulini, J. Russ, M. Sun, H. Vogel, I. Vorobiev, M. Weinberg

University of Colorado Boulder, Boulder, U.S.A.

J.P. Cumalat, W.T. Ford, F. Jensen, A. Johnson, M. Krohn, S. Leontsinis, T. Mulholland, K. Stenson, S.R. Wagner

Cornell University, Ithaca, U.S.A.

J. Alexander, J. Chaves, J. Chu, S. Dittmer, K. Mcdermott, N. Mirman, J.R. Patterson, A. Rinkevicius, A. Ryd, L. Skinnari, L. Soffi, S.M. Tan, Z. Tao, J. Thom, J. Tucker, P. Wittich, M. Zientek

Fermi National Accelerator Laboratory, Batavia, U.S.A.

S. Abdullin, M. Albrow, G. Apollinari, A. Apresyan, A. Apyan, S. Banerjee, L.A.T. Bauerdick, A. Beretvas, J. Berryhill, P.C. Bhat, G. Bolla, K. Burkett, J.N. Butler, A. Canepa, G.B. Cerati, H.W.K. Cheung, F. Chlebana, M. Cremonesi, J. Duarte, V.D. Elvira, J. Freeman, Z. Gecse, E. Gottschalk, L. Gray, D. Green, S. Grünendahl, O. Gutsche, R.M. Harris, S. Hasegawa, J. Hirschauer, Z. Hu, B. Jayatilaka, S. Jindariani, M. Johnson, U. Joshi, B. Klima, B. Kreis, S. Lammel, D. Lincoln, R. Lipton, M. Liu, T. Liu, R. Lopes De Sá, J. Lykken, K. Maeshima, N. Magini, J.M. Marraffino, S. Maruyama, D. Mason, P. McBride, P. Merkel, S. Mrenna, S. Nahn, V. O’Dell, K. Pedro, O. Prokofyev, G. Rakness, L. Ristori, B. Schneider, E. Sexton-Kennedy, A. Soha, W.J. Spalding, L. Spiegel, S. Stoynev, J. Strait, N. Strobbe, L. Taylor, S. Tkaczyk, N.V. Tran, L. Uplegger, E.W. Vaandering, C. Vernieri, M. Verzocchi, R. Vidal, M. Wang, H.A. Weber, A. Whitbeck

University of Florida, Gainesville, U.S.A.

D. Acosta, P. Avery, P. Bortignon, D. Bourilkov, A. Brinkerhoff, A. Carnes, M. Carver, D. Curry, S. Das, R.D. Field, I.K. Furic, J. Konigsberg, A. Korytov, K. Kotov, P. Ma, K. Matchev, H. Mei, G. Mitselmakher, D. Rank, D. Sperka, N. Terentyev, L. Thomas, J. Wang, S. Wang, J. Yelton

Florida International University, Miami, U.S.A.

Y.R. Joshi, S. Linn, P. Markowitz, J.L. Rodriguez

Florida State University, Tallahassee, U.S.A.

A. Ackert, T. Adams, A. Askew, S. Hagopian, V. Hagopian, K.F. Johnson, T. Kolberg, G. Martinez, T. Perry, H. Prosper, A. Saha, A. Santra, R. Yohay

Florida Institute of Technology, Melbourne, U.S.A.

M.M. Baarmand, V. Bhopatkar, S. Colafranceschi, M. Hohlmann, D. Noonan, T. Roy, F. Yumiceva 
University of Illinois at Chicago (UIC), Chicago, U.S.A.

M.R. Adams, L. Apanasevich, D. Berry, R.R. Betts, R. Cavanaugh, X. Chen, O. Evdokimov, C.E. Gerber, D.A. Hangal, D.J. Hofman, K. Jung, J. Kamin, I.D. Sandoval Gonzalez, M.B. Tonjes, H. Trauger, N. Varelas, H. Wang, Z. Wu, J. Zhang

The University of Iowa, Iowa City, U.S.A.

B. Bilki ${ }^{65}$, W. Clarida, K. Dilsiz ${ }^{66}$, S. Durgut, R.P. Gandrajula, M. Haytmyradov, V. Khristenko, J.-P. Merlo, H. Mermerkaya ${ }^{67}$, A. Mestvirishvili, A. Moeller, J. Nachtman, H. Ogul ${ }^{68}$, Y. Onel, F. Ozok ${ }^{69}$, A. Penzo, C. Snyder, E. Tiras, J. Wetzel, K. Yi

Johns Hopkins University, Baltimore, U.S.A.

B. Blumenfeld, A. Cocoros, N. Eminizer, D. Fehling, L. Feng, A.V. Gritsan, P. Maksimovic, J. Roskes, U. Sarica, M. Swartz, M. Xiao, C. You

The University of Kansas, Lawrence, U.S.A.

A. Al-bataineh, P. Baringer, A. Bean, S. Boren, J. Bowen, J. Castle, S. Khalil, A. Kropivnitskaya, D. Majumder, W. Mcbrayer, M. Murray, C. Royon, S. Sanders, E. Schmitz, R. Stringer, J.D. Tapia Takaki, Q. Wang

Kansas State University, Manhattan, U.S.A.

A. Ivanov, K. Kaadze, Y. Maravin, A. Mohammadi, L.K. Saini, N. Skhirtladze, S. Toda

Lawrence Livermore National Laboratory, Livermore, U.S.A.

F. Rebassoo, D. Wright

University of Maryland, College Park, U.S.A.

C. Anelli, A. Baden, O. Baron, A. Belloni, B. Calvert, S.C. Eno, C. Ferraioli, N.J. Hadley,

S. Jabeen, G.Y. Jeng, R.G. Kellogg, J. Kunkle, A.C. Mignerey, F. Ricci-Tam, Y.H. Shin,

A. Skuja, S.C. Tonwar

\section{Massachusetts Institute of Technology, Cambridge, U.S.A.}

D. Abercrombie, B. Allen, V. Azzolini, R. Barbieri, A. Baty, R. Bi, S. Brandt, W. Busza, I.A. Cali, M. D'Alfonso, Z. Demiragli, G. Gomez Ceballos, M. Goncharov, D. Hsu, Y. Iiyama, G.M. Innocenti, M. Klute, D. Kovalskyi, Y.S. Lai, Y.-J. Lee, A. Levin, P.D. Luckey, B. Maier, A.C. Marini, C. Mcginn, C. Mironov, S. Narayanan, X. Niu, C. Paus, C. Roland, G. Roland, J. Salfeld-Nebgen, G.S.F. Stephans, K. Tatar, D. Velicanu, J. Wang, T.W. Wang, B. Wyslouch

University of Minnesota, Minneapolis, U.S.A.

A.C. Benvenuti, R.M. Chatterjee, A. Evans, P. Hansen, S. Kalafut, Y. Kubota, Z. Lesko, J. Mans, S. Nourbakhsh, N. Ruckstuhl, R. Rusack, J. Turkewitz

University of Mississippi, Oxford, U.S.A.

J.G. Acosta, S. Oliveros

University of Nebraska-Lincoln, Lincoln, U.S.A.

E. Avdeeva, K. Bloom, D.R. Claes, C. Fangmeier, R. Gonzalez Suarez, R. Kamalieddin, I. Kravchenko, J. Monroy, J.E. Siado, G.R. Snow, B. Stieger 
State University of New York at Buffalo, Buffalo, U.S.A.

M. Alyari, J. Dolen, A. Godshalk, C. Harrington, I. Iashvili, D. Nguyen, A. Parker, S. Rappoccio, B. Roozbahani

Northeastern University, Boston, U.S.A.

G. Alverson, E. Barberis, A. Hortiangtham, A. Massironi, D.M. Morse, D. Nash, T. Orimoto, R. Teixeira De Lima, D. Trocino, D. Wood

Northwestern University, Evanston, U.S.A.

S. Bhattacharya, O. Charaf, K.A. Hahn, N. Mucia, N. Odell, B. Pollack, M.H. Schmitt, K. Sung, M. Trovato, M. Velasco

University of Notre Dame, Notre Dame, U.S.A.

N. Dev, M. Hildreth, K. Hurtado Anampa, C. Jessop, D.J. Karmgard, N. Kellams, K. Lannon, N. Loukas, N. Marinelli, F. Meng, C. Mueller, Y. Musienko ${ }^{34}$, M. Planer, A. Reinsvold, R. Ruchti, G. Smith, S. Taroni, M. Wayne, M. Wolf, A. Woodard

The Ohio State University, Columbus, U.S.A.

J. Alimena, L. Antonelli, B. Bylsma, L.S. Durkin, S. Flowers, B. Francis, A. Hart, C. Hill, W. Ji, B. Liu, W. Luo, D. Puigh, B.L. Winer, H.W. Wulsin

Princeton University, Princeton, U.S.A.

A. Benaglia, S. Cooperstein, O. Driga, P. Elmer, J. Hardenbrook, P. Hebda, S. Higginbotham, D. Lange, J. Luo, D. Marlow, K. Mei, I. Ojalvo, J. Olsen, C. Palmer, P. Piroué, D. Stickland, C. Tully

University of Puerto Rico, Mayaguez, U.S.A.

S. Malik, S. Norberg

Purdue University, West Lafayette, U.S.A.

A. Barker, V.E. Barnes, S. Folgueras, L. Gutay, M.K. Jha, M. Jones, A.W. Jung, A. Khatiwada, D.H. Miller, N. Neumeister, C.C. Peng, J.F. Schulte, J. Sun, F. Wang, W. Xie

Purdue University Northwest, Hammond, U.S.A.

T. Cheng, N. Parashar, J. Stupak

Rice University, Houston, U.S.A.

A. Adair, B. Akgun, Z. Chen, K.M. Ecklund, F.J.M. Geurts, M. Guilbaud, W. Li, B. Michlin, M. Northup, B.P. Padley, J. Roberts, J. Rorie, Z. Tu, J. Zabel

University of Rochester, Rochester, U.S.A.

A. Bodek, P. de Barbaro, R. Demina, Y.t. Duh, T. Ferbel, M. Galanti, A. Garcia-Bellido, J. Han, O. Hindrichs, A. Khukhunaishvili, K.H. Lo, P. Tan, M. Verzetti

The Rockefeller University, New York, U.S.A.

R. Ciesielski, K. Goulianos, C. Mesropian 
Rutgers, The State University of New Jersey, Piscataway, U.S.A.

A. Agapitos, J.P. Chou, Y. Gershtein, T.A. Gómez Espinosa, E. Halkiadakis, M. Heindl,

E. Hughes, S. Kaplan, R. Kunnawalkam Elayavalli, S. Kyriacou, A. Lath, R. Montalvo, K. Nash, M. Osherson, H. Saka, S. Salur, S. Schnetzer, D. Sheffield, S. Somalwar, R. Stone, S. Thomas, P. Thomassen, M. Walker

University of Tennessee, Knoxville, U.S.A.

A.G. Delannoy, M. Foerster, J. Heideman, G. Riley, K. Rose, S. Spanier, K. Thapa

Texas A\&M University, College Station, U.S.A.

O. Bouhali ${ }^{70}$, A. Castaneda Hernandez ${ }^{70}$, A. Celik, M. Dalchenko, M. De Mattia, A. Delgado, S. Dildick, R. Eusebi, J. Gilmore, T. Huang, T. Kamon ${ }^{71}$, R. Mueller, Y. Pakhotin, R. Patel, A. Perloff, L. Perniè, D. Rathjens, A. Safonov, A. Tatarinov, K.A. Ulmer

\section{Texas Tech University, Lubbock, U.S.A.}

N. Akchurin, J. Damgov, F. De Guio, P.R. Dudero, J. Faulkner, E. Gurpinar, S. Kunori, K. Lamichhane, S.W. Lee, T. Libeiro, T. Peltola, S. Undleeb, I. Volobouev, Z. Wang

Vanderbilt University, Nashville, U.S.A.

S. Greene, A. Gurrola, R. Janjam, W. Johns, C. Maguire, A. Melo, H. Ni, P. Sheldon, S. Tuo, J. Velkovska, Q. Xu

University of Virginia, Charlottesville, U.S.A.

M.W. Arenton, P. Barria, B. Cox, R. Hirosky, A. Ledovskoy, H. Li, C. Neu, T. Sinthuprasith, X. Sun, Y. Wang, E. Wolfe, F. Xia

Wayne State University, Detroit, U.S.A.

R. Harr, P.E. Karchin, J. Sturdy, S. Zaleski

University of Wisconsin - Madison, Madison, WI, U.S.A.

M. Brodski, J. Buchanan, C. Caillol, S. Dasu, L. Dodd, S. Duric, B. Gomber, M. Grothe, M. Herndon, A. Hervé, U. Hussain, P. Klabbers, A. Lanaro, A. Levine, K. Long, R. Loveless, G.A. Pierro, G. Polese, T. Ruggles, A. Savin, N. Smith, W.H. Smith, D. Taylor, N. Woods

$\dagger$ : Deceased

1: Also at Vienna University of Technology, Vienna, Austria

2: Also at State Key Laboratory of Nuclear Physics and Technology, Peking University, Beijing, China

3: Also at Universidade Estadual de Campinas, Campinas, Brazil

4: Also at Universidade Federal de Pelotas, Pelotas, Brazil

5: Also at Université Libre de Bruxelles, Bruxelles, Belgium

6: Also at Institute for Theoretical and Experimental Physics, Moscow, Russia

7: Also at Joint Institute for Nuclear Research, Dubna, Russia

8: Now at Cairo University, Cairo, Egypt

9: Also at Zewail City of Science and Technology, Zewail, Egypt

10: Also at Université de Haute Alsace, Mulhouse, France 
11: Also at Skobeltsyn Institute of Nuclear Physics, Lomonosov Moscow State University, Moscow, Russia

12: Also at Tbilisi State University, Tbilisi, Georgia

13: Also at CERN, European Organization for Nuclear Research, Geneva, Switzerland

14: Also at RWTH Aachen University, III. Physikalisches Institut A, Aachen, Germany

15: Also at University of Hamburg, Hamburg, Germany

16: Also at Brandenburg University of Technology, Cottbus, Germany

17: Also at Institute of Nuclear Research ATOMKI, Debrecen, Hungary

18: Also at MTA-ELTE Lendület CMS Particle and Nuclear Physics Group, Eötvös Loránd University, Budapest, Hungary

19: Also at Institute of Physics, University of Debrecen, Debrecen, Hungary

20: Also at Indian Institute of Technology Bhubaneswar, Bhubaneswar, India

21: Also at Institute of Physics, Bhubaneswar, India

22: Also at University of Visva-Bharati, Santiniketan, India

23: Also at University of Ruhuna, Matara, Sri Lanka

24: Also at Isfahan University of Technology, Isfahan, Iran

25: Also at Yazd University, Yazd, Iran

26: Also at Plasma Physics Research Center, Science and Research Branch, Islamic Azad University, Tehran, Iran

27: Also at Università degli Studi di Siena, Siena, Italy

28: Also at INFN Sezione di Milano-Bicocca; Università di Milano-Bicocca, Milano, Italy

29: Also at Purdue University, West Lafayette, U.S.A.

30: Also at International Islamic University of Malaysia, Kuala Lumpur, Malaysia

31: Also at Malaysian Nuclear Agency, MOSTI, Kajang, Malaysia

32: Also at Consejo Nacional de Ciencia y Tecnología, Mexico city, Mexico

33: Also at Warsaw University of Technology, Institute of Electronic Systems, Warsaw, Poland

34: Also at Institute for Nuclear Research, Moscow, Russia

35: Now at National Research Nuclear University 'Moscow Engineering Physics Institute' (MEPhI), Moscow, Russia

36: Also at St. Petersburg State Polytechnical University, St. Petersburg, Russia

37: Also at University of Florida, Gainesville, U.S.A.

38: Also at P.N. Lebedev Physical Institute, Moscow, Russia

39: Also at California Institute of Technology, Pasadena, U.S.A.

40: Also at Budker Institute of Nuclear Physics, Novosibirsk, Russia

41: Also at Faculty of Physics, University of Belgrade, Belgrade, Serbia

42: Also at INFN Sezione di Roma; Sapienza Università di Roma, Rome, Italy

43: Also at University of Belgrade, Faculty of Physics and Vinca Institute of Nuclear Sciences, Belgrade, Serbia

44: Also at Scuola Normale e Sezione dell'INFN, Pisa, Italy

45: Also at National and Kapodistrian University of Athens, Athens, Greece

46: Also at Riga Technical University, Riga, Latvia

47: Also at Universität Zürich, Zurich, Switzerland

48: Also at Stefan Meyer Institute for Subatomic Physics (SMI), Vienna, Austria

49: Also at Istanbul University, Faculty of Science, Istanbul, Turkey

50: Also at Gaziosmanpasa University, Tokat, Turkey

51: Also at Istanbul Aydin University, Istanbul, Turkey

52: Also at Mersin University, Mersin, Turkey

53: Also at Cag University, Mersin, Turkey 
54: Also at Piri Reis University, Istanbul, Turkey

55: Also at Adiyaman University, Adiyaman, Turkey

56: Also at Izmir Institute of Technology, Izmir, Turkey

57: Also at Necmettin Erbakan University, Konya, Turkey

58: Also at Marmara University, Istanbul, Turkey

59: Also at Kafkas University, Kars, Turkey

60: Also at Istanbul Bilgi University, Istanbul, Turkey

61: Also at Rutherford Appleton Laboratory, Didcot, United Kingdom

62: Also at School of Physics and Astronomy, University of Southampton, Southampton, United Kingdom

63: Also at Instituto de Astrofísica de Canarias, La Laguna, Spain

64: Also at Utah Valley University, Orem, U.S.A.

65: Also at BEYKENT UNIVERSITY, Istanbul, Turkey

66: Also at Bingol University, Bingol, Turkey

67: Also at Erzincan University, Erzincan, Turkey

68: Also at Sinop University, Sinop, Turkey

69: Also at Mimar Sinan University, Istanbul, Istanbul, Turkey

70: Also at Texas A\&M University at Qatar, Doha, Qatar

71: Also at Kyungpook National University, Daegu, Korea 